ఠ

\title{
Aging brain: the effect of combined cognitive and physical training on cognition as compared to cognitive and physical training alone $-\mathrm{a}$ systematic review
}

This article was published in the following Dove Press journal:

Clinical Interventions in Aging

\section{Clémence Joubert \\ Hanna Chainay}

Laboratory for the Study of Cognitive Mechanisms, University of Lyon 2, Lyon, France
Correspondence: Clémence Joubert Laboratoire d'Etude des Mécanismes Cognitifs, Université Lyon 2, 5, Avenue Pierre Mendès France, 69676 Bron Cedex, France

Email clemence.joubert@univ-lyon2.fr

\begin{abstract}
This review presents a critical examination of current knowledge of the impact of combined cognitive and physical training on cognition in healthy elderly subjects. The objectives are to evaluate the contribution of cognitive and physical training to the enhancement of cognition, and to determine the interest of combining these two training types in one intervention in terms of the benefits for cognition (direct and transfer), long-term maintenance, and transfer to daily living. To do so, a systematic electronic search was conducted in PubMed and Google Scholar. Exclusion criteria were animal and pathological aging studies. We focused on the shared and different behavioral impacts of these two types of training on cognition, as well as their functional and structural impact on the brain. The review indicates that both cognitive and physical training have an impact on cognition and on the brain. However, each type of training seems to preferentially enhance different cognitive functions and specifically impact both brain structure and function. Even though some results argue in favor of a complementarity between cognitive and physical training and the superiority of combined cognitive and physical training, the current state of knowledge does not permit any definitive conclusion. Thus, the present review indicates the need for additional investigations.
\end{abstract}

Keywords: cognitive training, physical training, combined cognitive and physical training, healthy adults

\section{Introduction}

Western society is facing a significant increase of the elderly population. Individuals are living even longer and are confronted with disability and fragility. ${ }^{1-3}$ Even though majority of the elderly have relatively well-preserved health, $20 \%$ of older adults aged 70 years or more experience difficulties in their everyday activities and lose their independence. ${ }^{4,5}$ It is well established that normal aging induces anatomo-physiological changes in the brain that impact some aspects of cognition, ${ }^{6}$ and particularly speed of processing, working memory, and executive functions. ${ }^{7,8}$ This decline is mostly due to a dysfunction of the pre-frontal cortex, which is especially vulnerable and thus becomes prematurely atrophied in normal aging. ${ }^{9}$ Some authors have shown that in the face of this atrophy, the aging brain reorganizes its functioning, for example, in terms of the hemispheric lateralization of the solicited regions. Indeed, the Hemispheric Asymmetry Reduction in Older Adults (HAROLD) ${ }^{10}$ model holds that for any given cognitive task, older adults exhibit bilateral brain activation, whereas young adults exhibit unilateral activation to achieve the same performance. For instance, it has been 
found that when young and older adults had to perform an episodic memory task, although both groups succeeded in the task, the older adults exhibited a bilateral recruitment of pre-frontal regions, whereas the young adults exhibited unilateral recruitment of these same regions. ${ }^{10}$ Two hypotheses can explain this phenomenon. The compensatory hypothesis postulates that older adults would compensate for the difficulties they face by additionally recruiting contralateral regions to contribute to the ongoing task. ${ }^{10,11}$ The dedifferentiation hypothesis postulates that asymmetry reduction results from difficulties in recruiting specific cerebral regions that are required for a given task, thus leading to a more generalized recruitment. ${ }^{12}$ Based on this idea, some authors proposed the Compensation Related Utilization of Neural Circuits Hypothesis (CRUNCH) model, ${ }^{11}$ in which they explained that for a given task, older adults need more neuronal resources than young adults, thus resulting in this over-recruitment of brain regions in older adults. ${ }^{13}$ The HAROLD and CRUNCH models provide relevant explanations about how the aging brain adapts and organizes its functioning in response to the age-related decline in cognition. However, the Scaffolding Theory of Aging and Cognition (revised-version - STAC-r) ${ }^{7}$ proposes a more complete approach to aging cognition.

STAC-r not only takes into consideration age-related cognitive decline and its negative impact on the brain and cognition, but also positive aspects that can counteract and delay brain neurodegeneration in normal aging. In fact, many factors can influence cognition through life in both positive (high levels of education, social interaction, intellectual stimulation, physical activity) and negative (low level of education, lack of physical activity, health problems, alcohol or drug abuse) ways. ${ }^{14}$ The STAC model suggests that as people age, there is an adaptation and a reorganization of brain functioning. More precisely, brain deterioration due to age-related decline results in a response in the form of a scaffolding of new compensatory networks, depending on the factors that positively and negatively influence cognition. STAC-r is the first model to explicitly include the concept of behavioral interventions, such as cognitive and physical training, while also modeling cognitive and cerebral aging, and to consider that training contributes to the scaffolding of new neuronal networks. In a more general way, this model proposes that having a rich cognitive and physical environment throughout life helps to preserve brain function. ${ }^{7}$

Behavioral interventions that aim to protect brain function against age-related decline are often described in terms of cognitive training (particularly oriented toward memory and executive functions) $)^{15,16}$ and physical training (especially aerobics). ${ }^{17}$ The question addressed by this review is whether it is relevant to combine cognitive training and physical training in one training intervention in order to improve the training outcomes as compared to each of these training modes administered alone.

\section{Methods}

\section{Search strategy}

A systematic electronic search was conducted in PubMed and Google Scholar with the following keywords: cognitive training, physical training, combined cognitive and physical training (CCPT), working memory, executive function, cognitive enhancement, endurance, aerobics, walking, everyday activities, elderly, and healthy older adults. The research was restricted to the period from January 2000 to November 2017 and to articles written in English. The search was also complemented by references to articles and "related articles".

\section{Selection process}

We only considered empirical studies that used a training intervention designed for healthy older adults. Animal studies, studies of persons with cognitive impairments, or studies that did not use a training design were excluded. A total of 52 studies are included in this review: 32 relating to single cognitive training, 10 relating to single physical training, and 11 relating both to cognitive and physical training. Among these 11 last studies, three are based on a direct comparison between cognitive and physical training alone and eight studies addressed CCPT.

\section{Evaluation of methodological quality}

We performed a qualitative evaluation of the 52 studies that were included in the review. We used the Physiotherapy Evidence Database (PEDro) scale. ${ }^{18}$ This scale contains 11 criteria that evaluate the methodological quality of studies. Each fulfilled criterion is worth 1 point, and nonfulfilled criterion is worth 0 point. Total score between 9 and 11 points means a good methodological quality, total score between 6 and 8 means medium methodological quality, and total score of less than 6 points means low methodological quality. Tables 1-3 report levels of methodological quality of the studies that used, respectively, cognitive training alone, physical training alone, and cognitive and physical training together.

In the following sections, we will first present the impact of cognitive and physical training on cognition, and brain structure and function. Then, the differential 


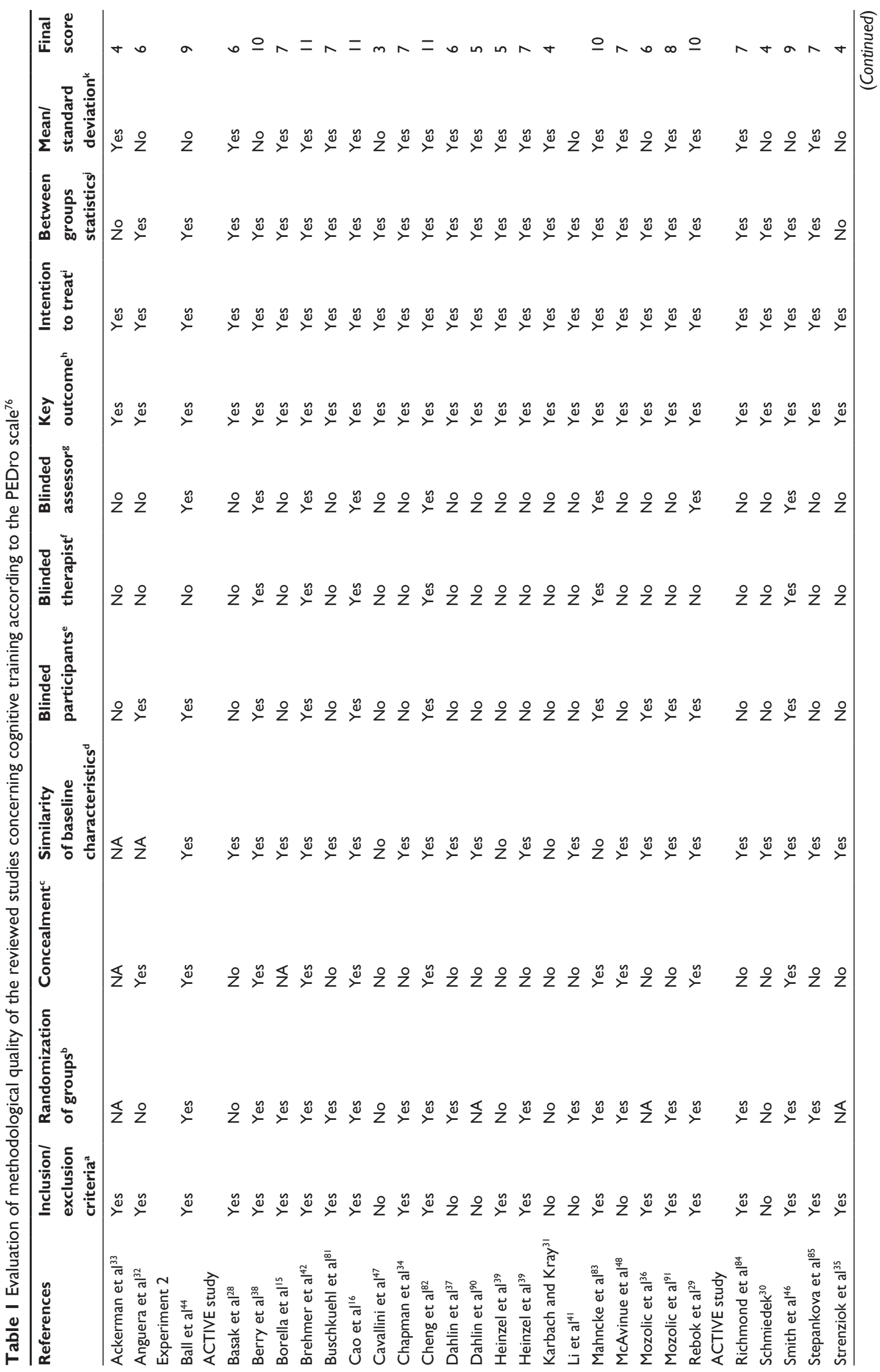



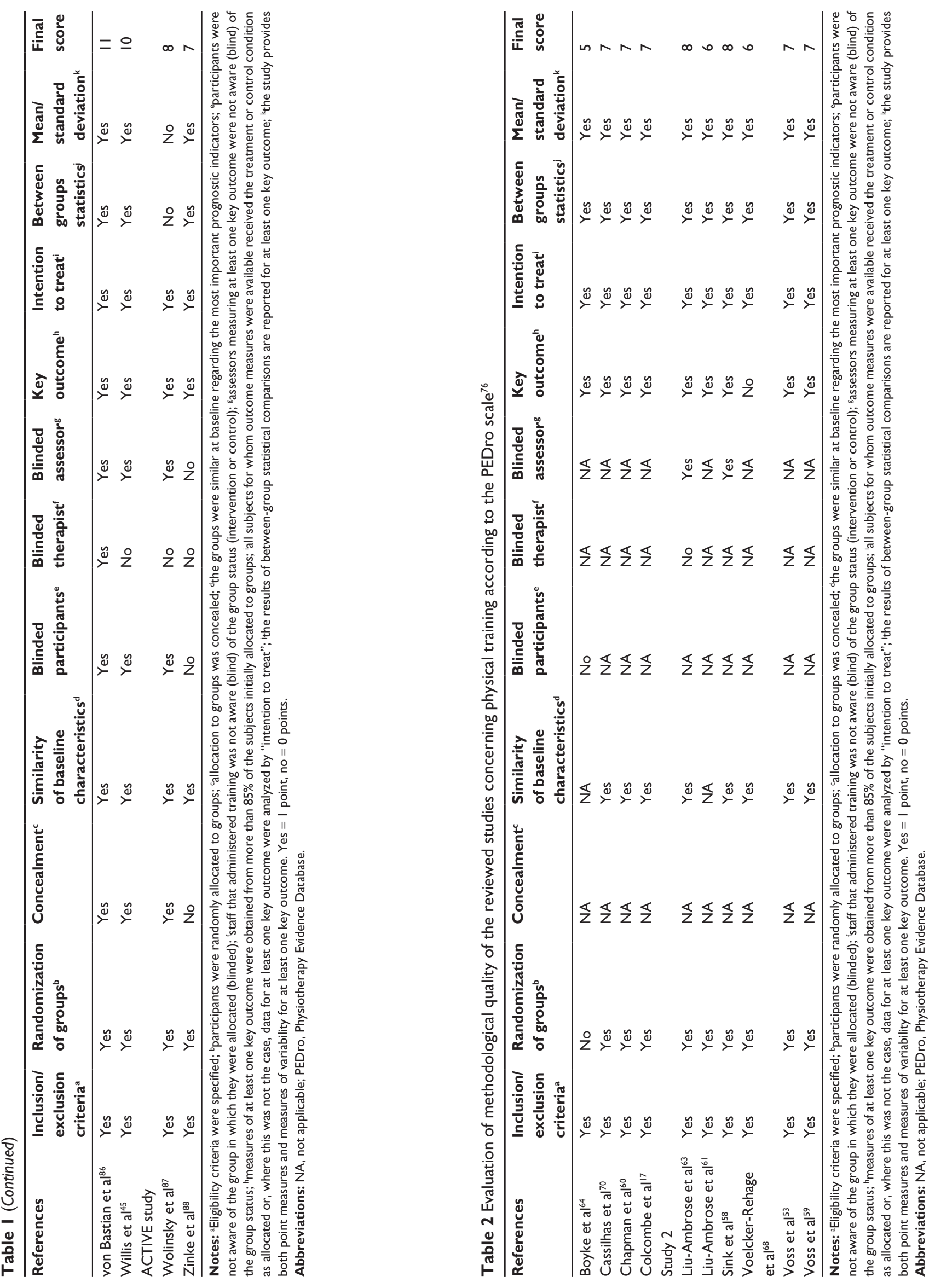


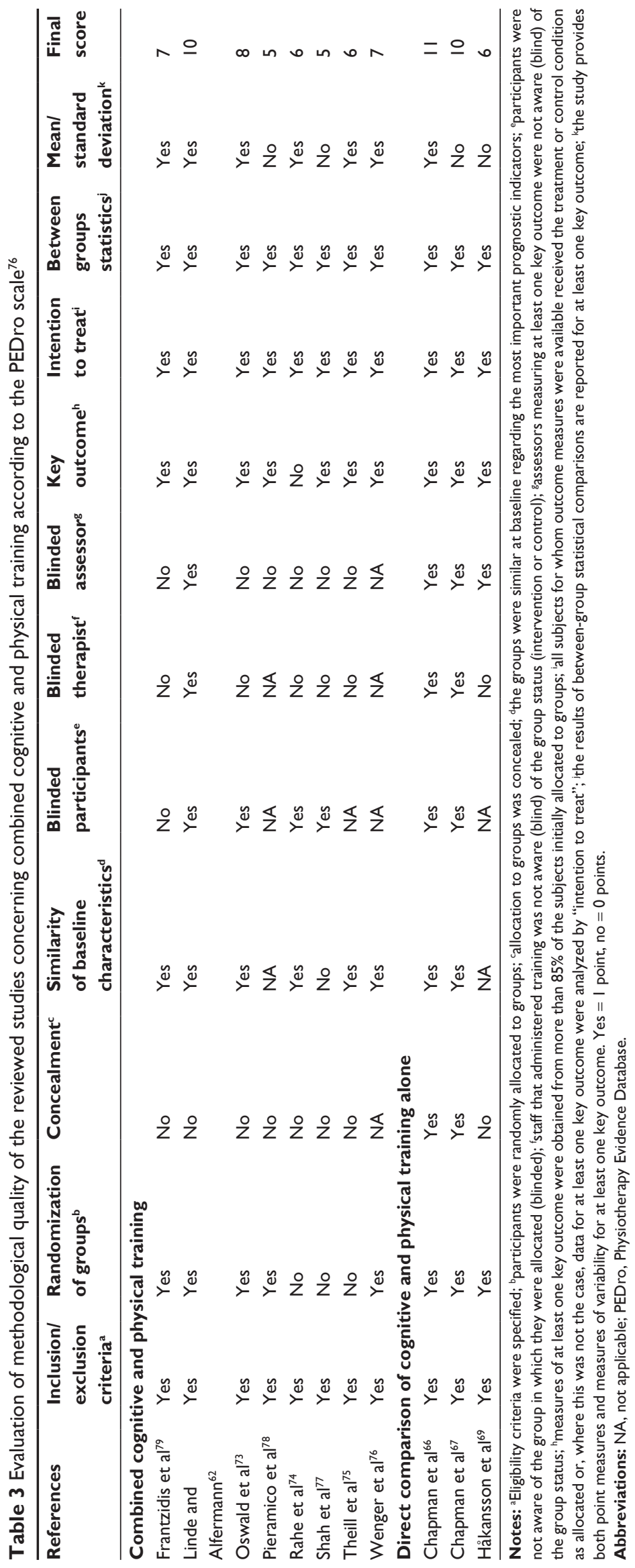


and complementary effects of CCPT will be addressed. Tables 4-6 present details concerning the procedure and results of each cited study in this review, respectively, for cognitive training alone, physical training alone, and cognitive and physical training together. In the tables, we only reported statistically significant (ie, $p<0.05$ ) outcomes that are described as increase or decrease compared to baseline. Giving the irregularity in report of effect size in the selected studies, we did not include this data in the tables. However, globally, the effect sizes (reported as $\eta^{2}$ ) are in the range between 0.001 and 0.88 in studies concerning cognitive training alone, between 0.01 and 0.97 in studies concerning physical training alone, between 0.14 and 0.34 in studies concerning CCPT, and between 0.001 and 0.37 in studies that report direct comparison between cognitive and physical training alone.

\section{Specific impact of cognitive and physical training on cognition, and brain structure and function}

Several studies have shown the benefits of either cognitive (Table 4) or physical (Table 5) training on older adults' brain and cognition. Some models of aging consider that brain structure and function adapt and reorganize due to cognitive and physical training (STAC-R), ${ }^{19}$ meaning that this non-pharmacological method induces plasticity in the aging brain. Cognitive and physical training are both said to improve and support cognitive functions and to delay neurodegenerative processes. Although both cognitive and physical training seem to have an impact on cognition and brain, each type of training has specific effects that are inherent to their respective natures. Below, we present the impact, first, of cognitive training and, second, of physical training on brain structure and function, and on cognition. Different meta-analysis and systematic reviews of these studies are available ${ }^{20-25}$ and our purpose is not to exhaustively address all points that have been already discussed in these reviews. We focused especially on transfer to untrained tasks and to everyday life and on maintenance.

\section{Cognitive training}

In the present review, we focus on cognitive training, although other types of cognitive interventions ${ }^{26}$ are used to improve cognition (eg, cognitive stimulation, cognitive rehabilitation). Cognitive training is based on the assumption that the aging brain and cognition can be improved through training (neuroplasticity) and uses repetitive exercises (laboratory made or commercial software such as Brain Aging,
Big Brain Academy, and Brain Challenge) targeted at specific cognitive functions, such as memory, attention, or executive function. One or more functions can be targeted during one and the same training intervention ${ }^{27}$ and the training can be performed individually or in small groups, although the work remains individual and there is no interaction with other people. The tasks are usually performed on a computer but pencil-and-paper exercises can also be proposed. On the behavioral level, exposure to cognitive training is supposed to improve performance of cognitive tasks and older adults' everyday life. On the brain level, it is thought to induce functional and structural changes that help to reduce cognitive decline. These training-related changes are measured by 1 ) cortical thickness and gray matter volume reflecting quantity and state of neurons, 2) integrity of the white matter reflecting structural connectivity, and 3) neuronal activity and functional connectivity in specific networks in the resting condition (default mode network or dorsal attention network) or in task-specific conditions.

All the studies of cognitive training cited in the present review are presented in Table 4. Globally, most of the studies that used appropriate, controlled intervention designs have shown beneficial effects (statistically significant differences between trained and control group, and pre-test and post-test) of cognitive training in older adults on performance in cognitive tasks (trained or untrained) and/or on brain function and structure. Improvements after training with laboratory tasks have, in particular, been found for working memory, ${ }^{15,28}$ speed of processing, ${ }^{29,30}$ and executive functions. ${ }^{31,32}$ Some of the studies addressed the issue of using commercial brain training games, ${ }^{33}$ and also showed a positive impact on older adults' cognition. Studies investigating changes on brain level are less frequent. Among the 32 studies listed in Table 4, only eight studies investigated the impact of cognitive training on brain structure and function. Four studies measured white matter integrity or connectivity, ${ }^{16,25,34,39}$ two studies measured gray matter volume, ${ }^{36,39}$ two studies measured cerebral blood flow (CBF), ${ }^{34,36}$ and three studies measured functional activity. ${ }^{32,37,38}$ The main positive structural changes have been reported after single or multi-domain training in white matter in frontal, ${ }^{16}$ fronto-temporal, ${ }^{34}$ occipitotemporal, ${ }^{35}$ and occipital ${ }^{16}$ regions. Functional changes, expressed by 1) changes in $\mathrm{CBF},{ }^{34,36}$ 2) changes in functional connectivity, ${ }^{16,35} 3$ ) increase in task-related blood oxygen level dependent responses, ${ }^{37} 4$ ) increase of theta power, ${ }^{32}$ and 5) decrease in N100 amplitude, ${ }^{38}$ were reported. These functional changes occurred, in particular, in frontal, parietal, and occipital regions and in subcortical regions 


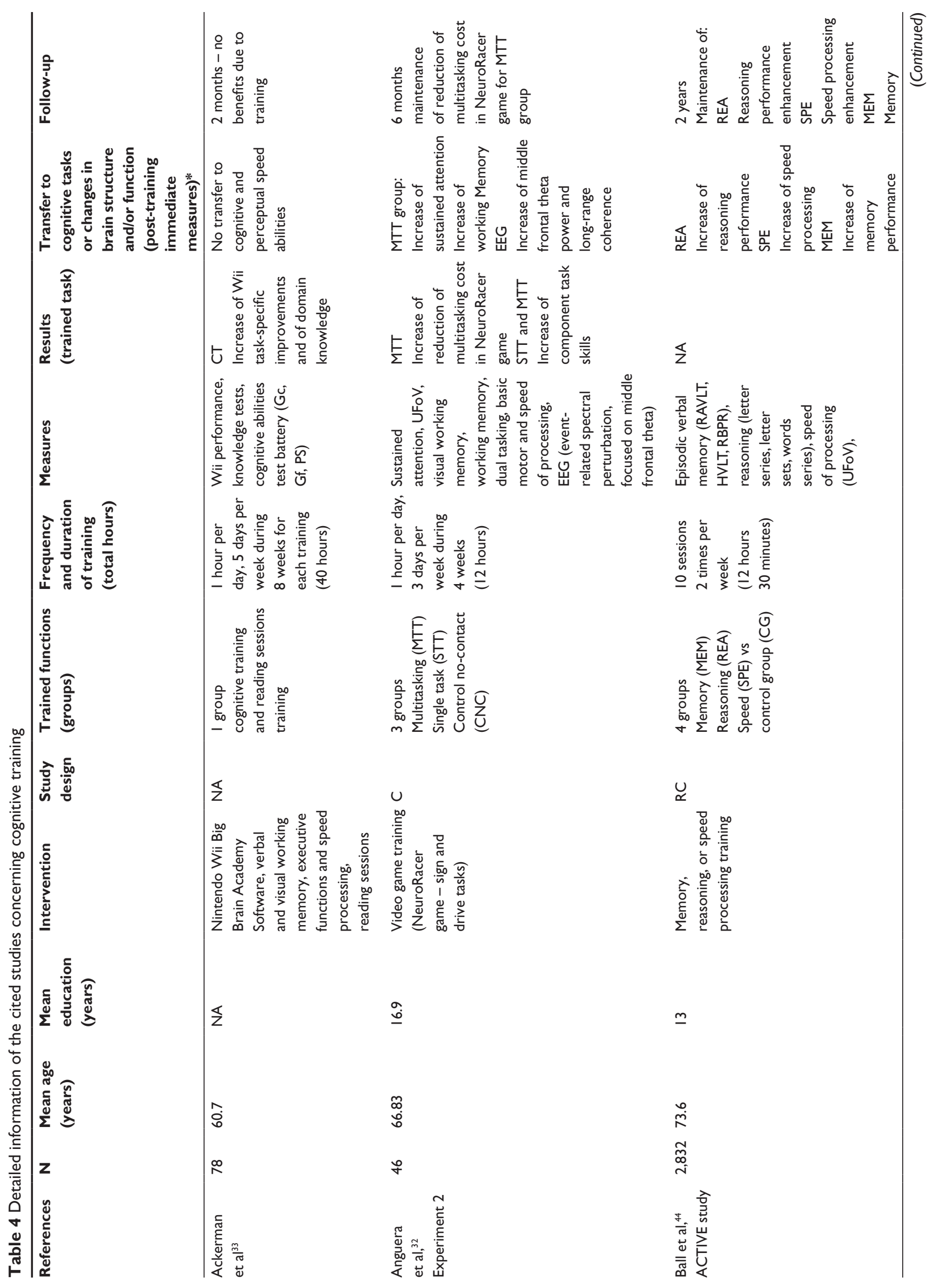




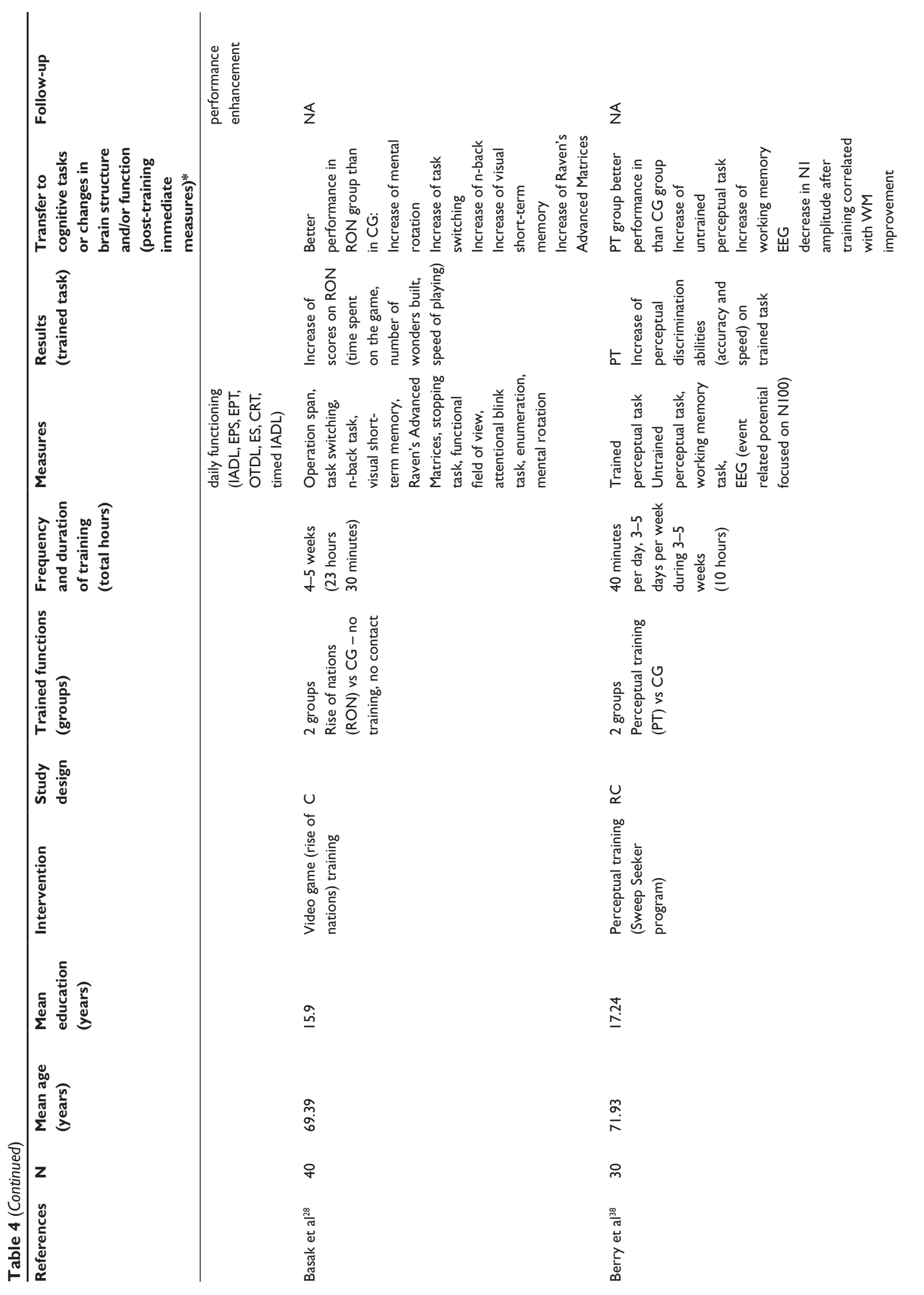



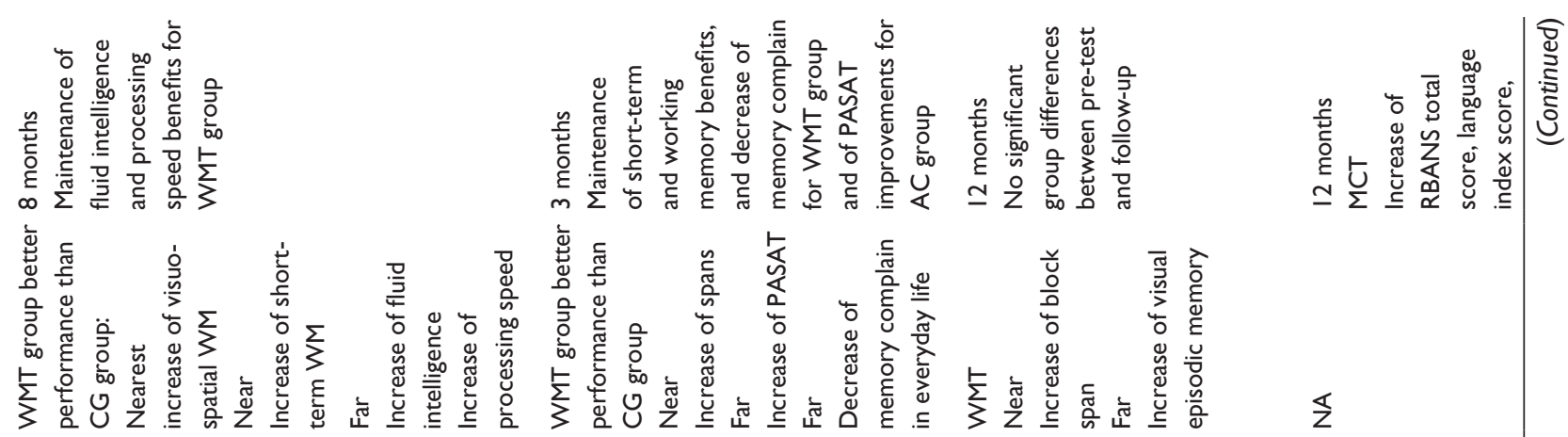

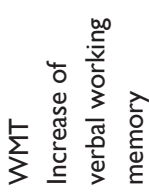

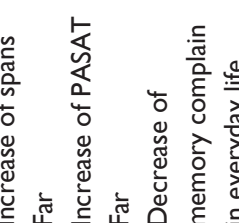

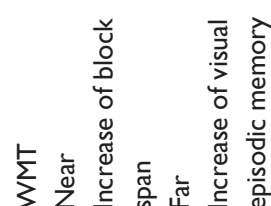

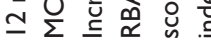
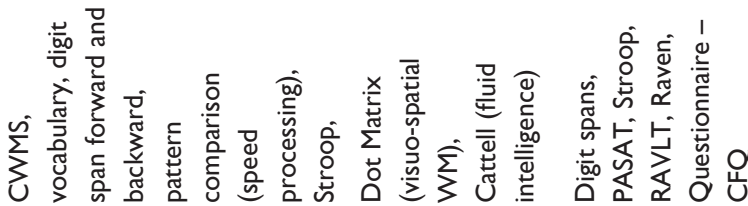

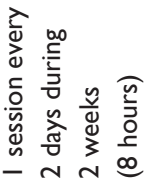

竞

U

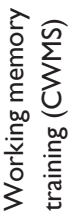

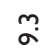

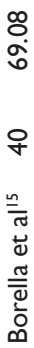

$\stackrel{m}{\underline{m}}$

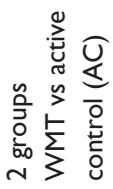

$\underset{\Upsilon}{\cup}$

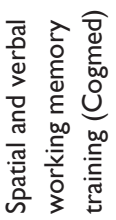

$\stackrel{\infty}{\text { ஸ் }}$

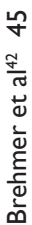

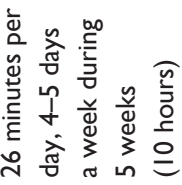

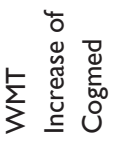

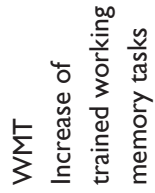

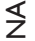

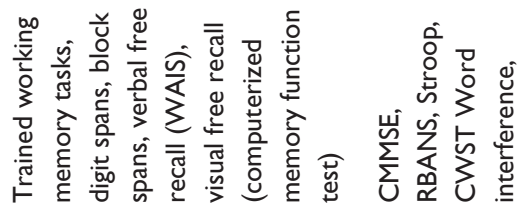

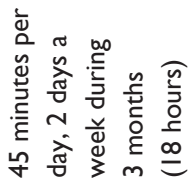

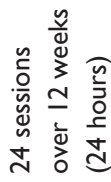

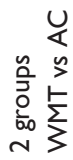

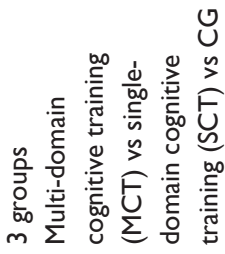

U

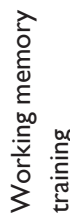

Ч

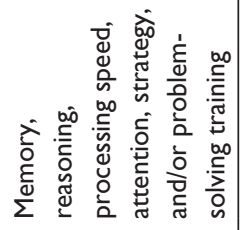

そ 으

$\infty \quad \bar{\circ}$

产

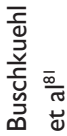

|




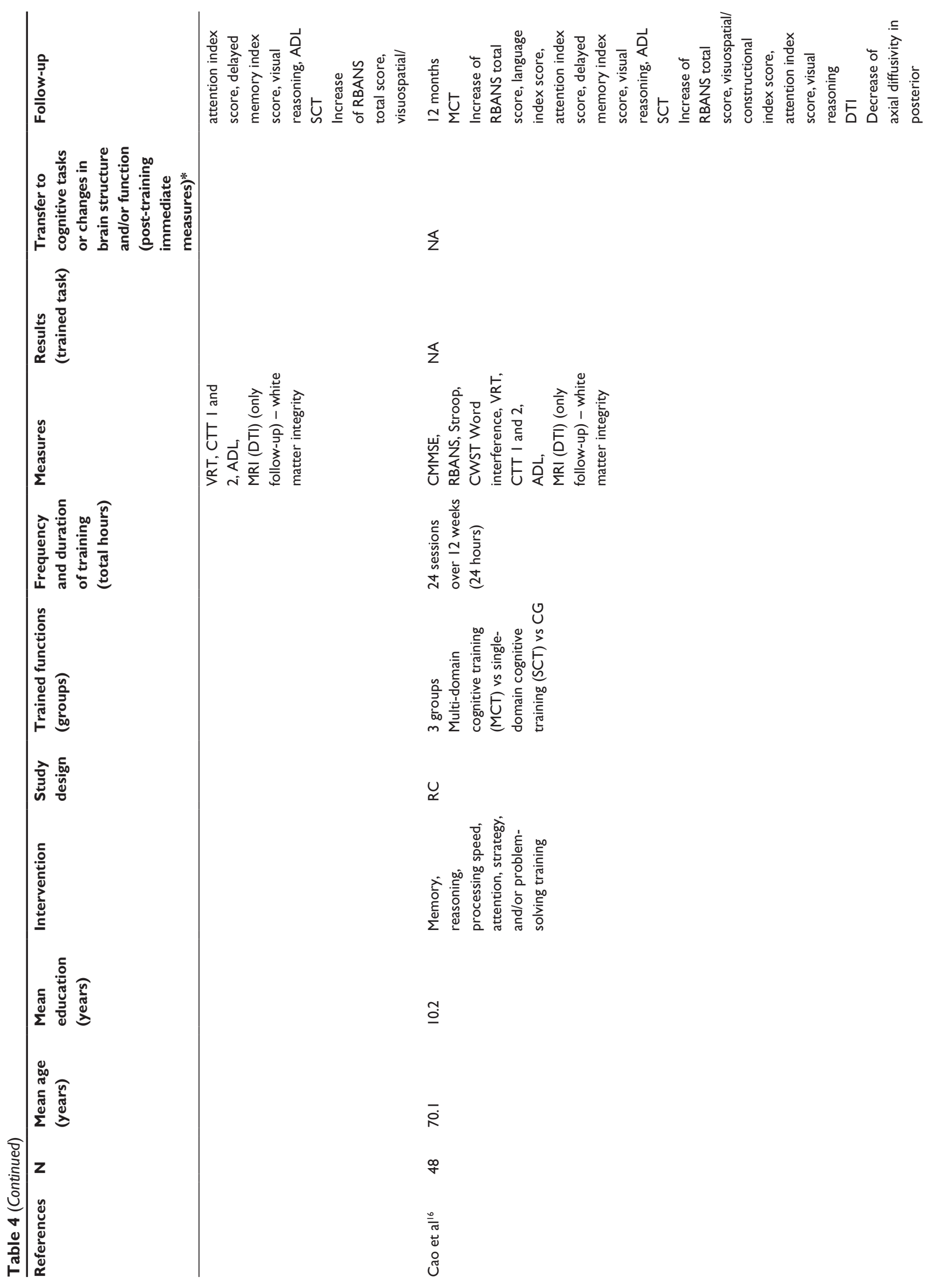



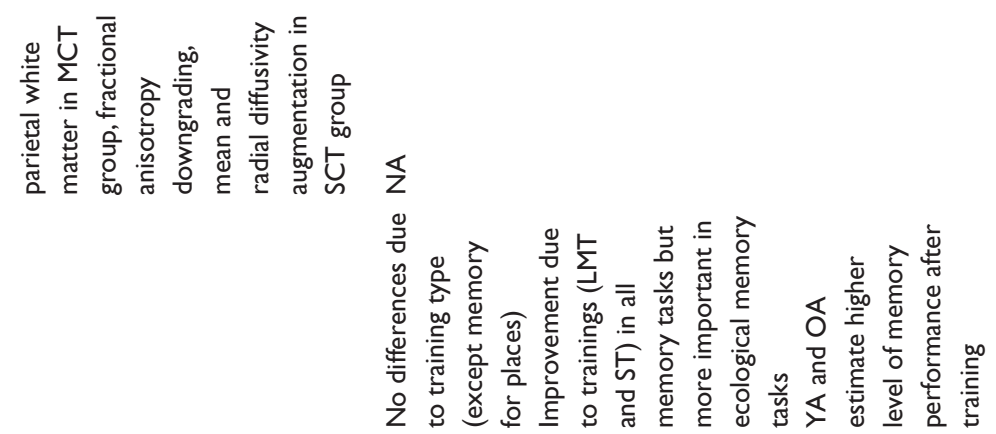

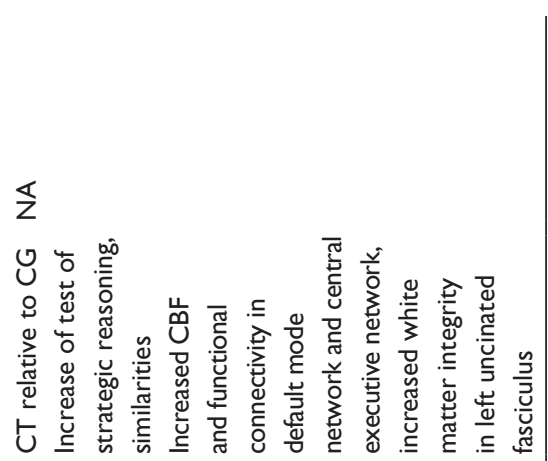

|

草

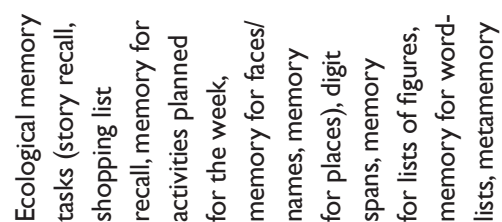

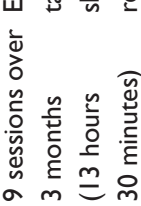

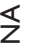
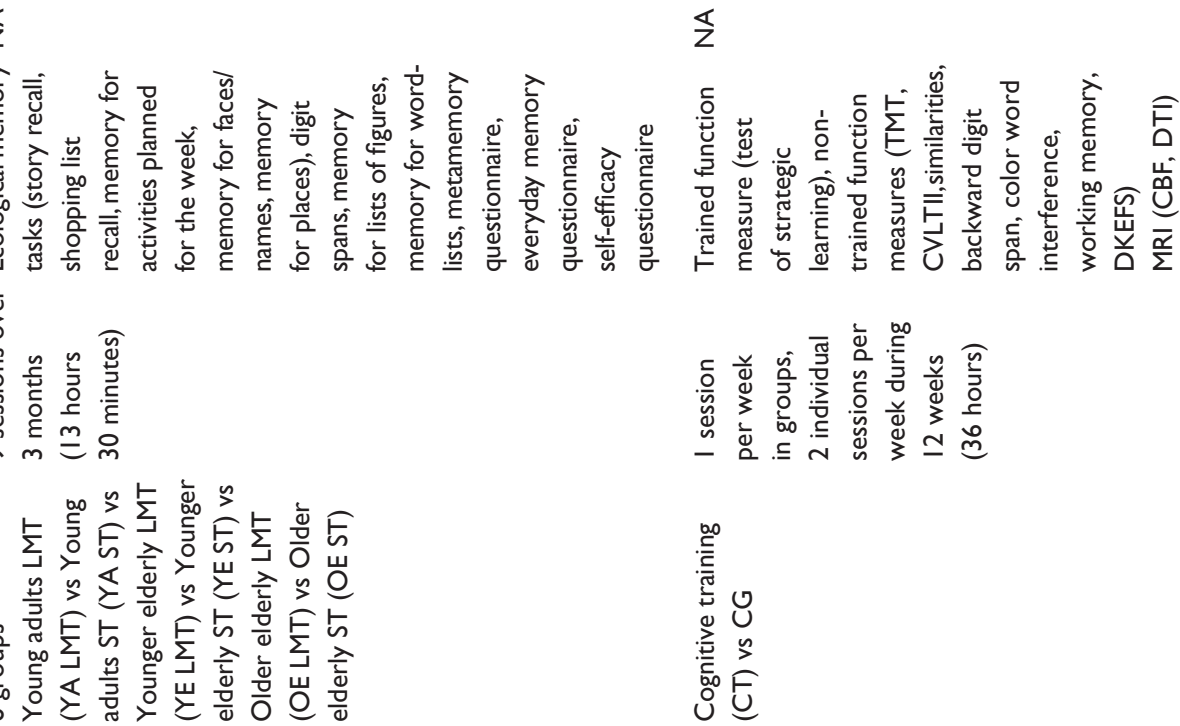

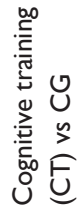

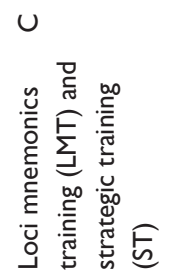

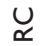

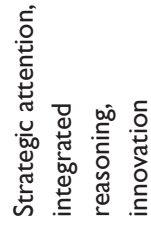

$\S$

$\S$

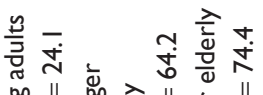

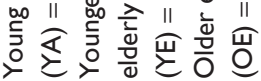

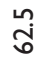

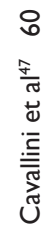

$\hat{m}$

䍃 


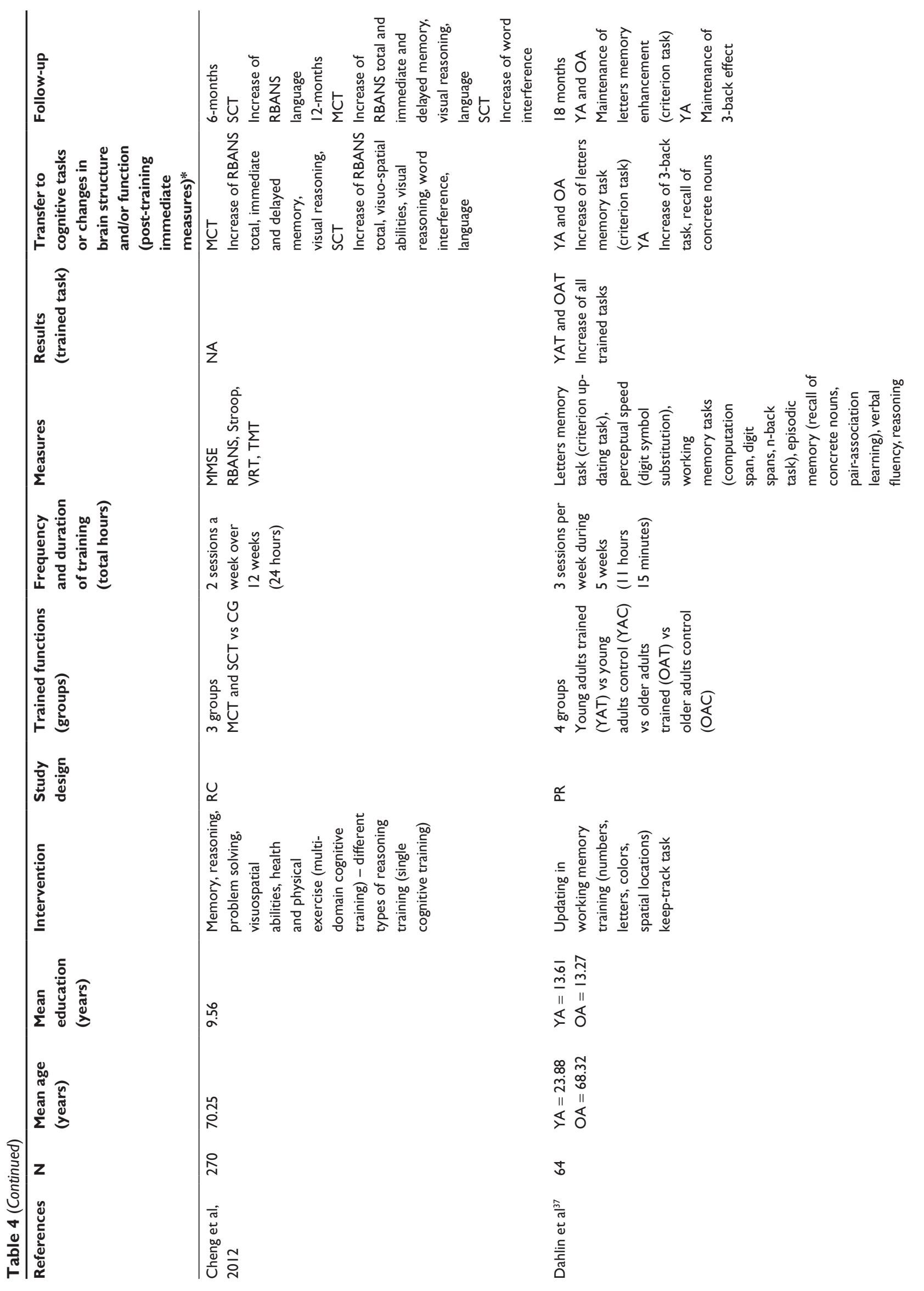




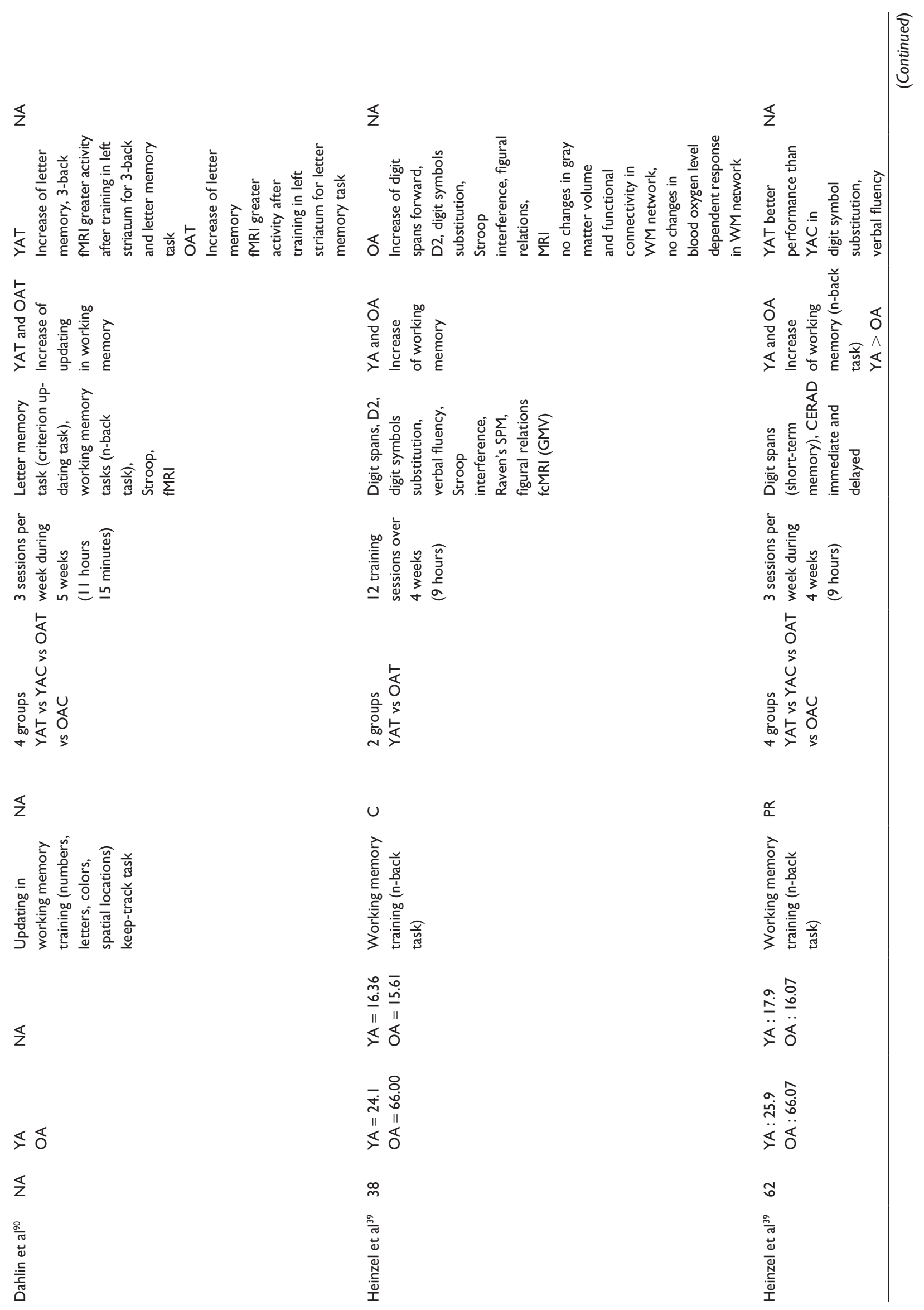




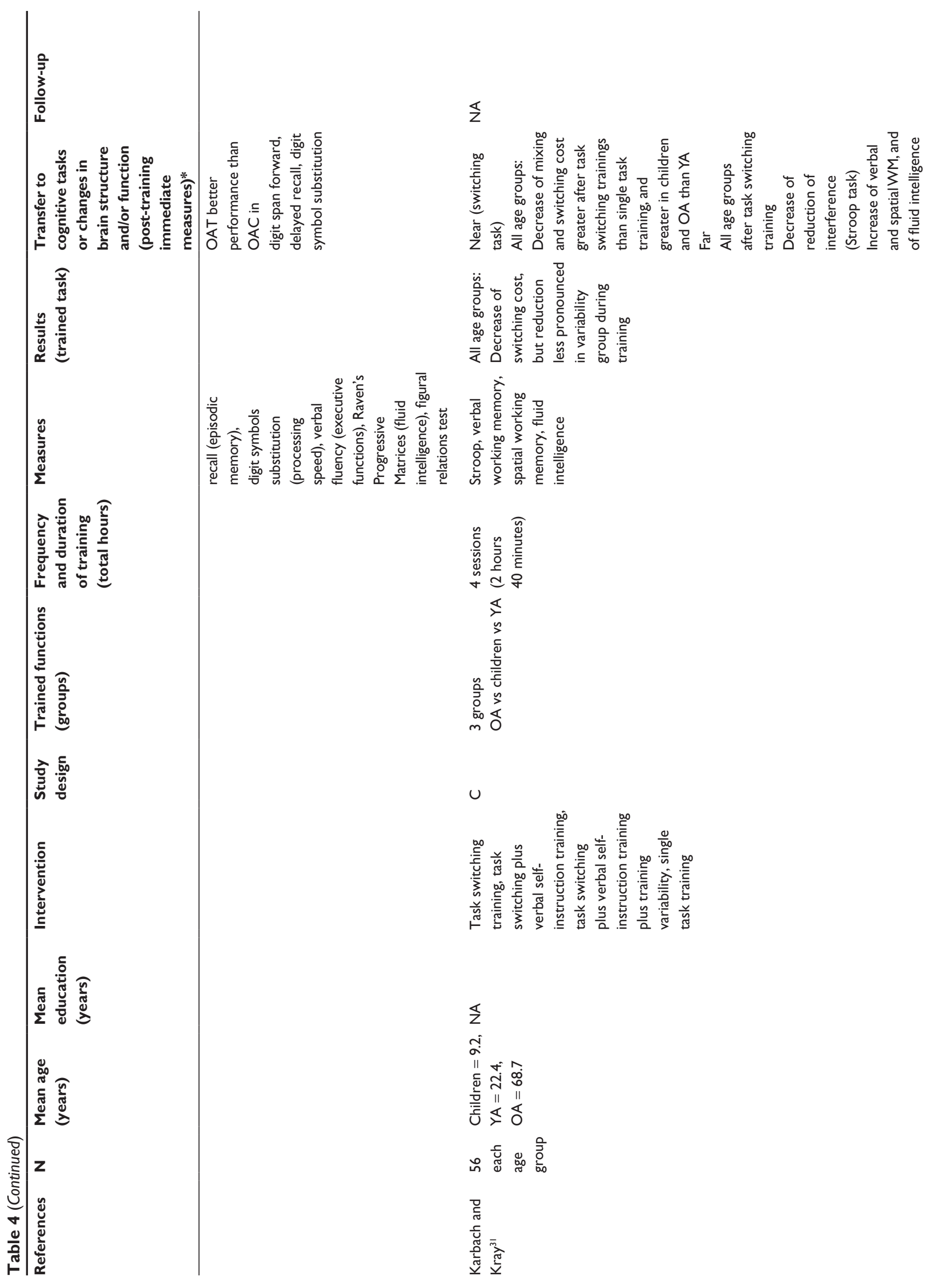




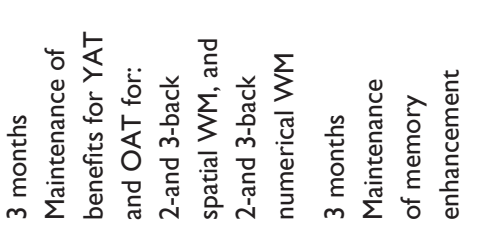

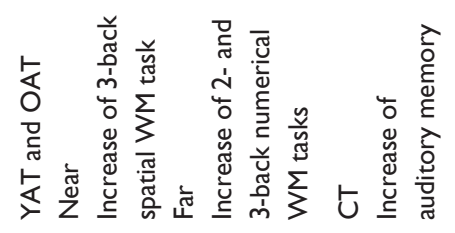

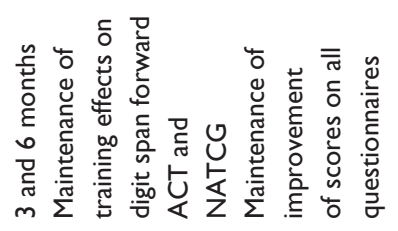

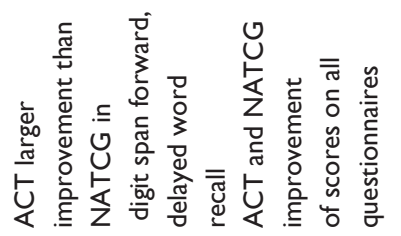

$\S$

¿

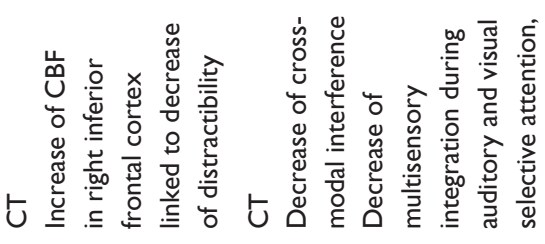

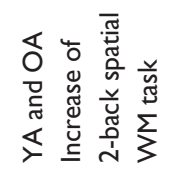

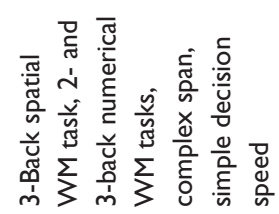

\section{त्वे ते}

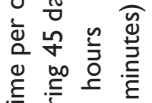

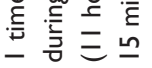

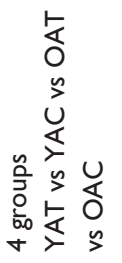

움

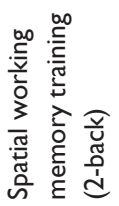

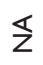

$\stackrel{n}{\infty} \underset{n}{\infty}$

แ่

$\stackrel{4}{\longleftarrow}$

$\widehat{\infty}$

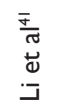

产

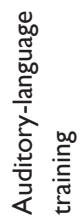

$\stackrel{m}{\underline{m}}$

’े

$\cong$

$\frac{\pi}{\pi}$

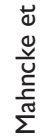

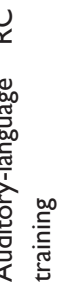

$\stackrel{n}{0}$

s. $\stackrel{s}{z}$

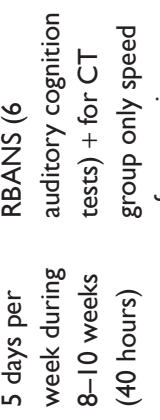

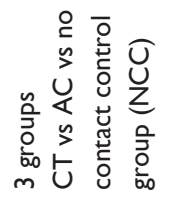

$\stackrel{n}{\gtrless}$

$\stackrel{m}{m}$

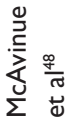

Ч

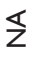

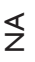

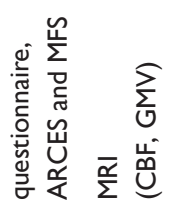

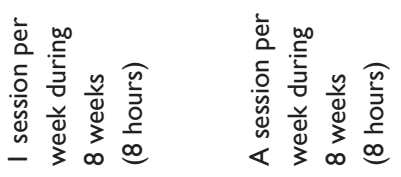

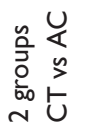
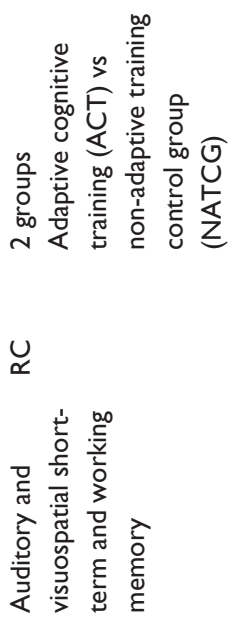

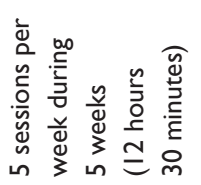

品它总

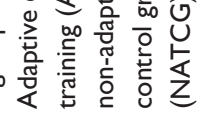

乏

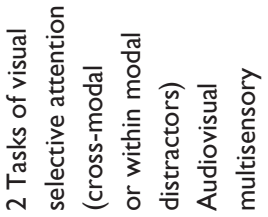

$\stackrel{\underline{n}}{\underline{\underline{n}}}$

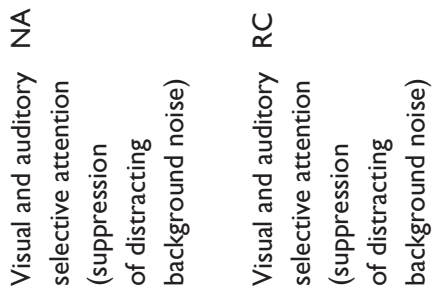

$\stackrel{\infty}{\underline{\infty}}$

के

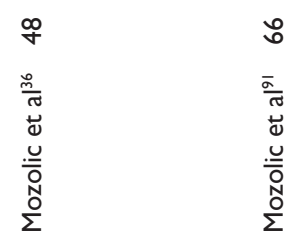




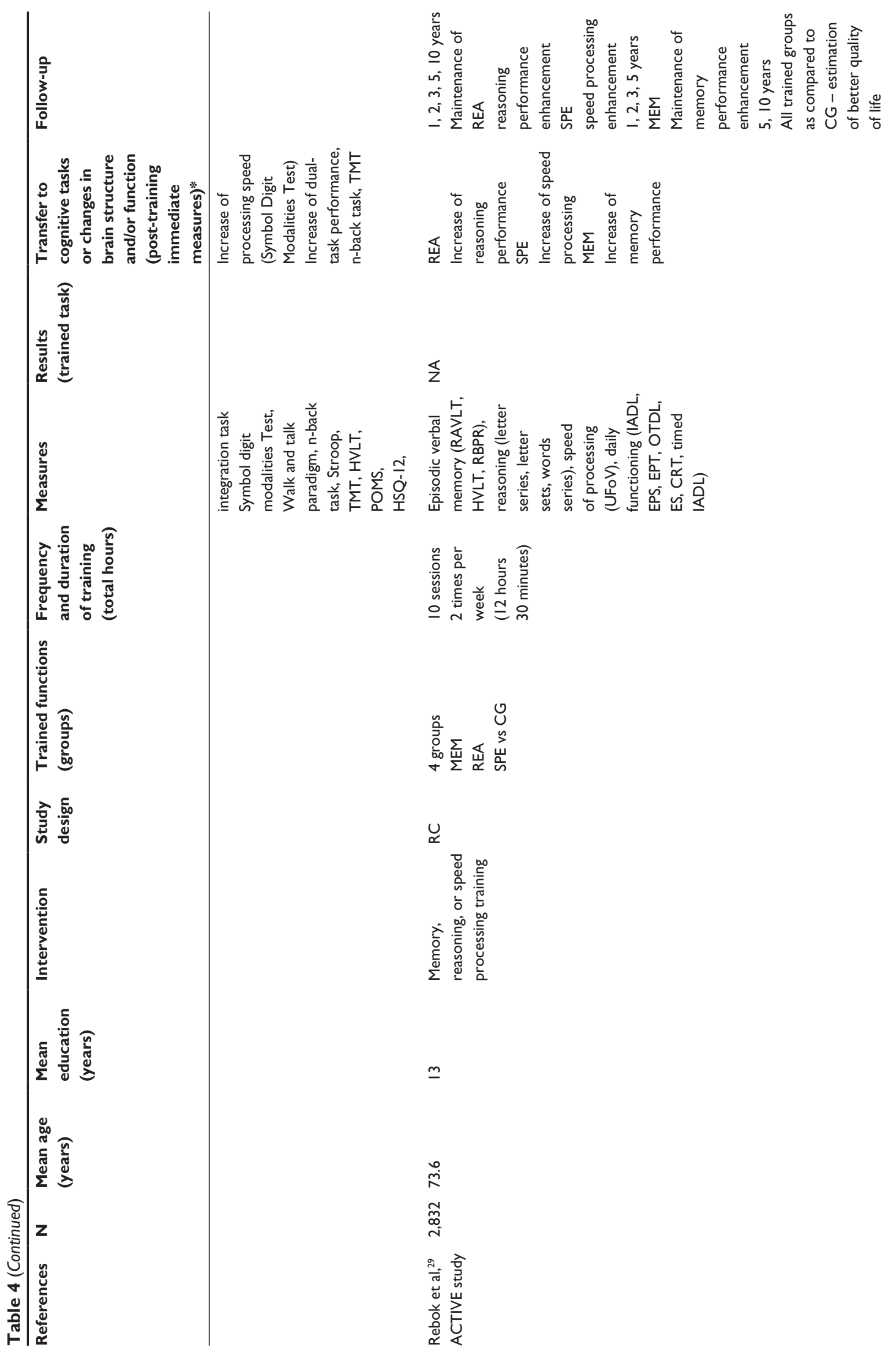




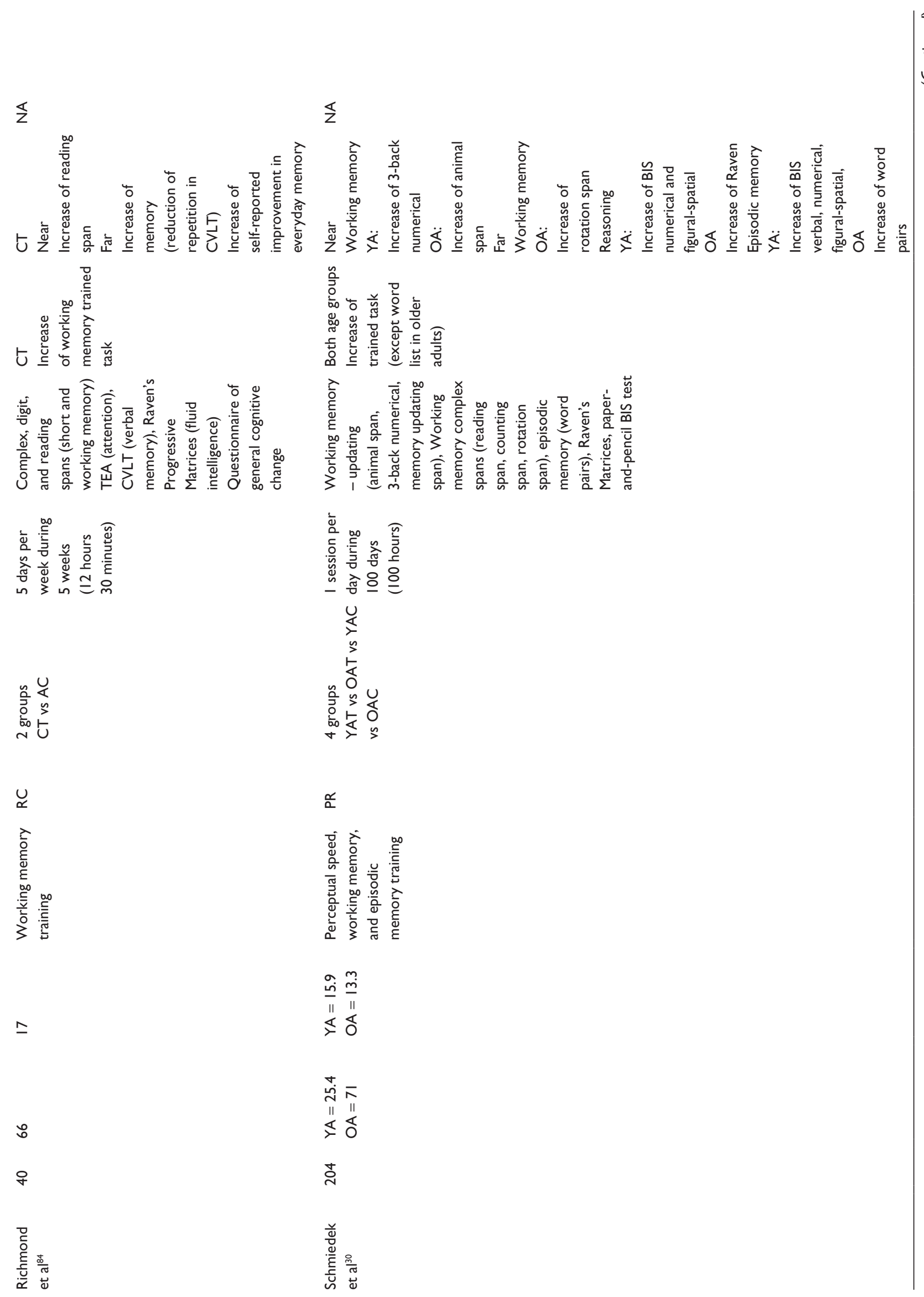




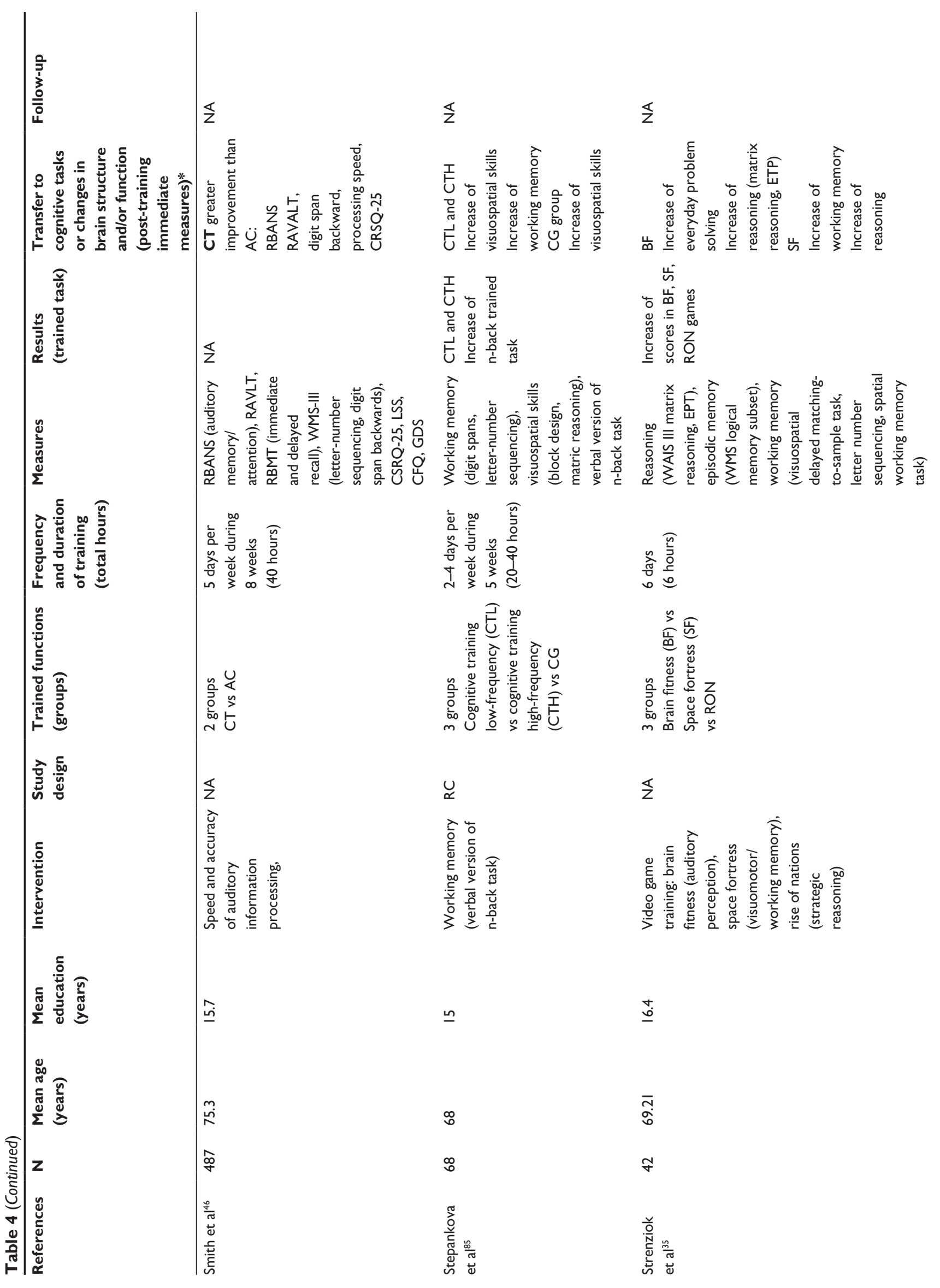




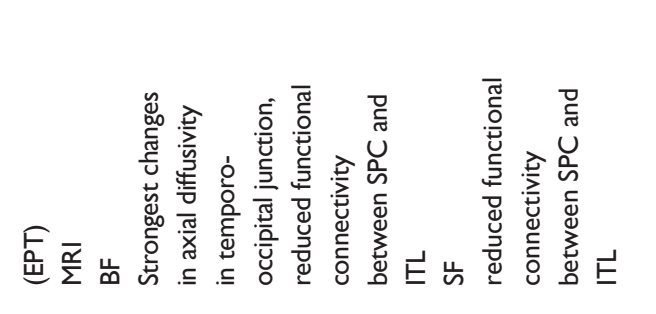

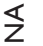

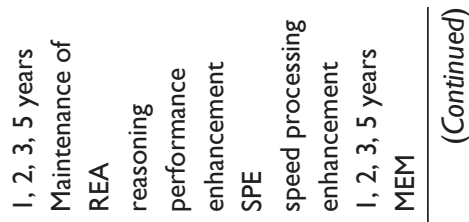

$\frac{\widehat{F}}{\frac{\sigma}{e}}$

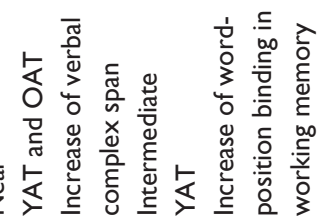

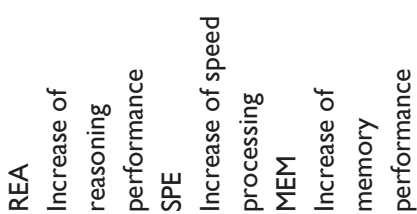

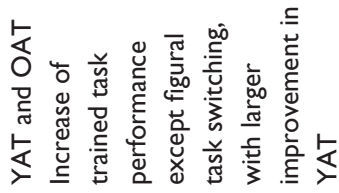

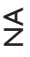

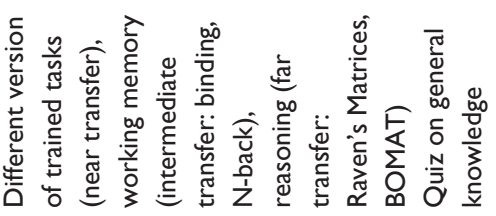

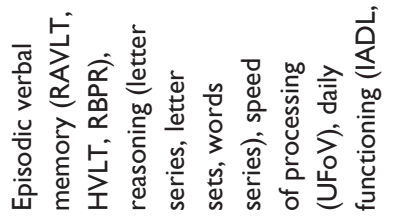

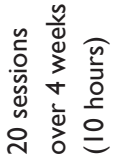

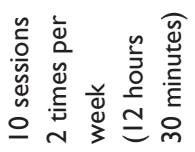

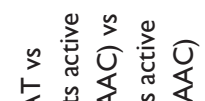

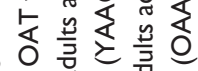

䒓

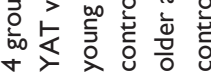

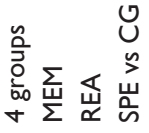

움

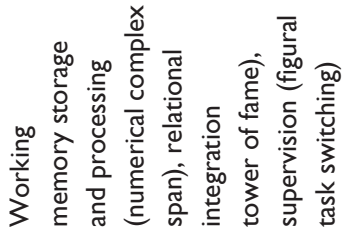

Y

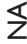

$\underline{m}$

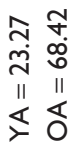

$\stackrel{\text { mi }}{\pi}$

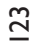

$\underset{\infty}{\infty}$

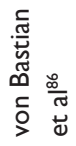

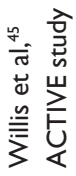




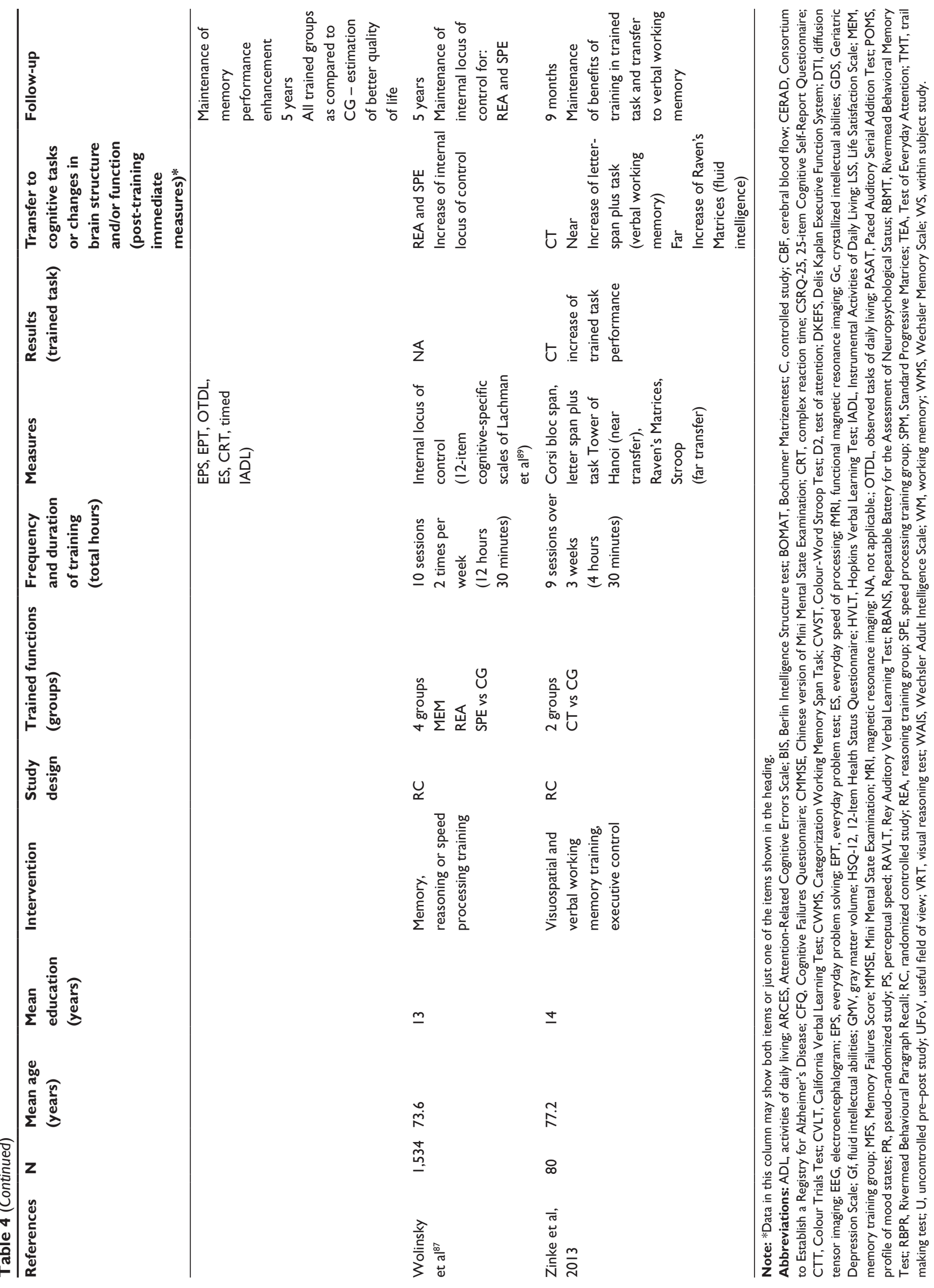




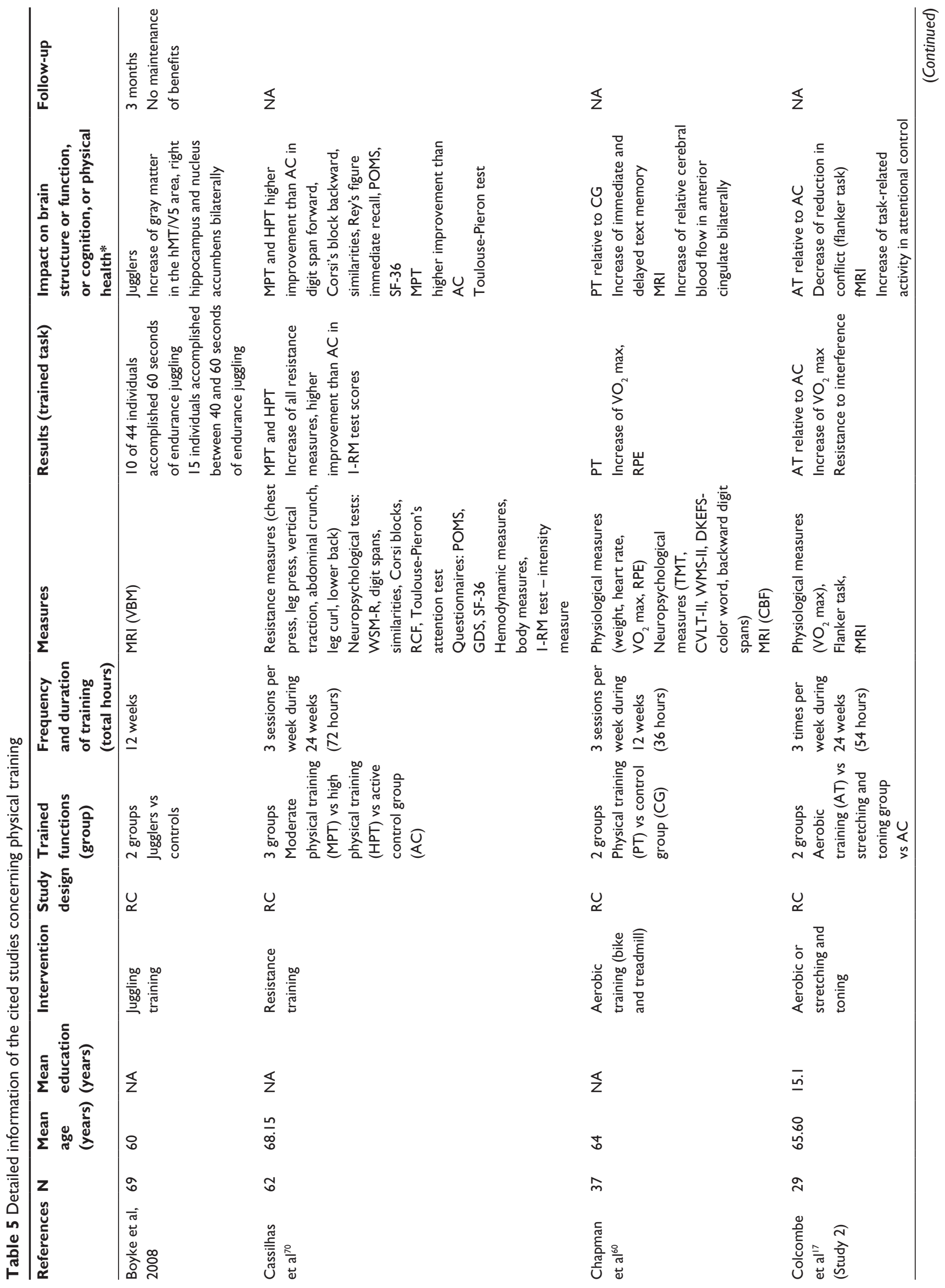




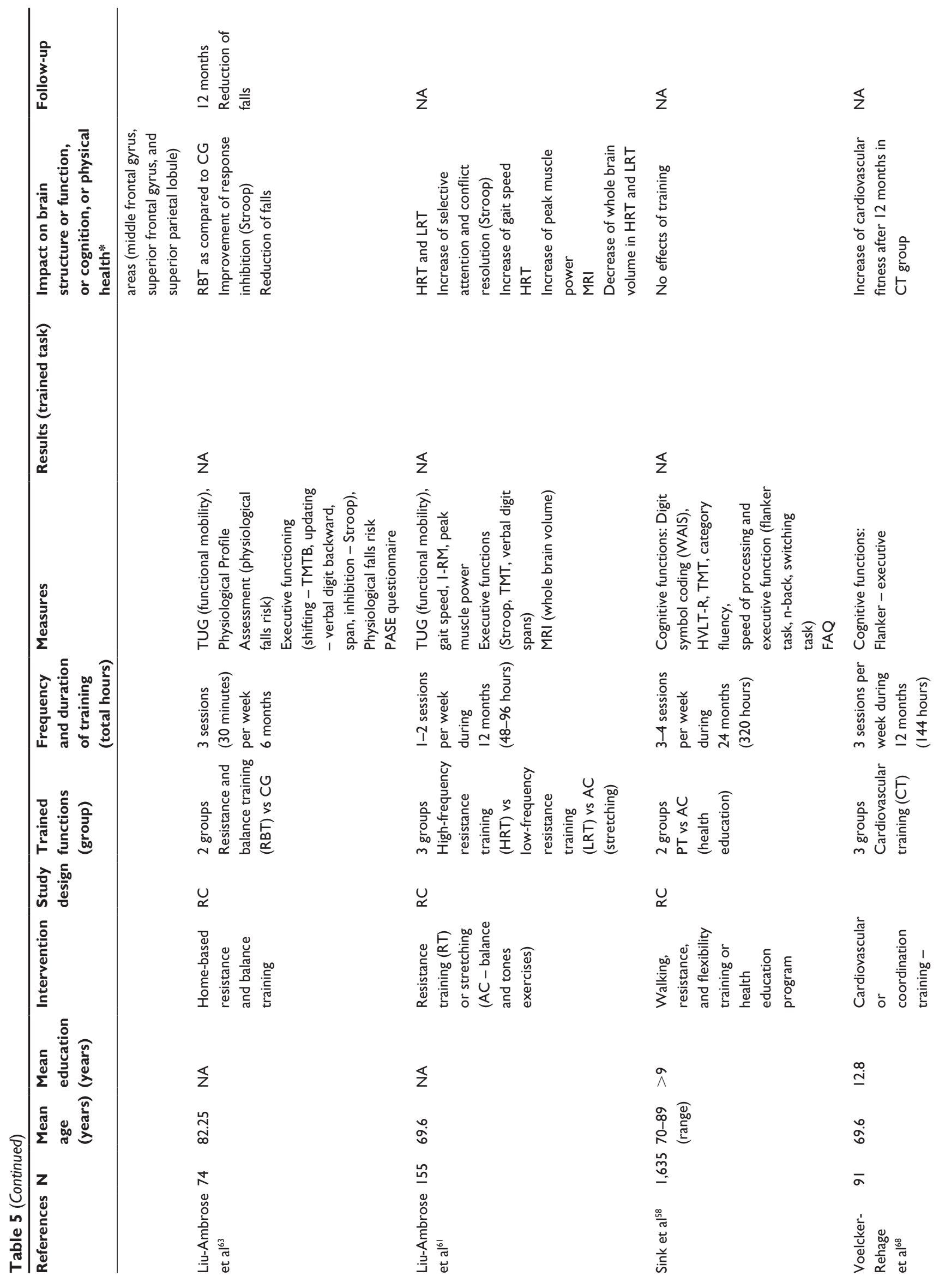




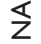

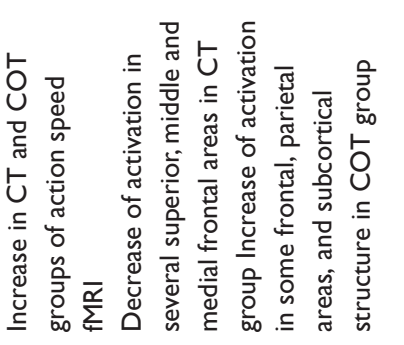

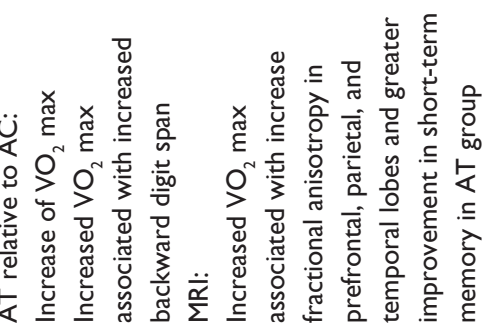

$\S$

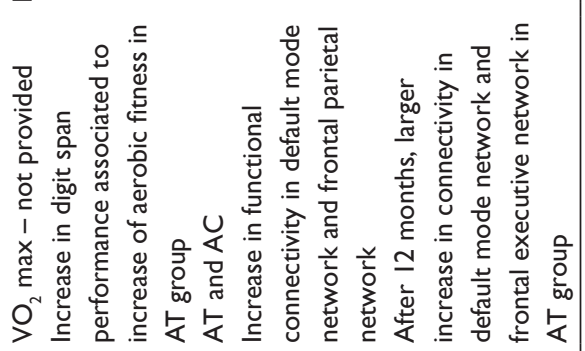

$\stackrel{s}{z}$

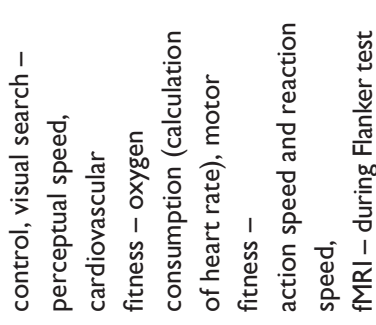

$\overleftarrow{z}$

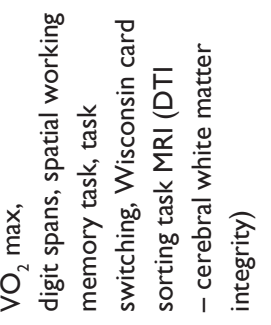

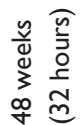

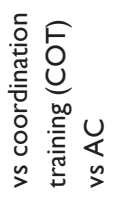

足

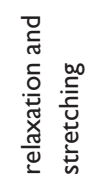

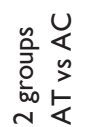

$\underset{\longleftarrow}{\longleftarrow}$

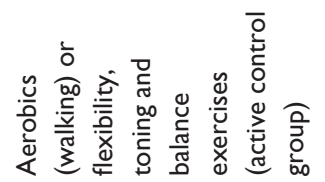

$\underline{\bar{\theta}}$

我

\&

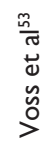

$\stackrel{s}{z}$

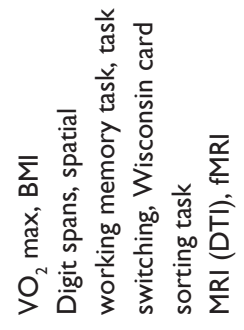

$\tilde{v}$
$\stackrel{y}{d}$
3
$\infty$
$\dot{y}$

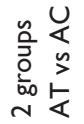

Ч

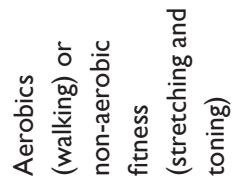

옴

选芒

萑

के

นำ

㠃

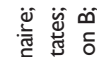

음 잉

Oे

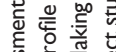

岛地 证

唹

울

害它方

Oे 巳

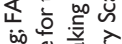

尊卷

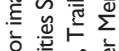

运密占

음 월

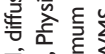

岁岁

岁通.

令

웜

文交要

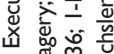

들

ज्ञ 후

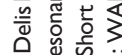

点造

落岕

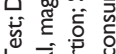

这金焉

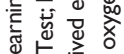

可 号

ว 造

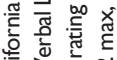

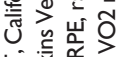

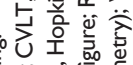

专范它

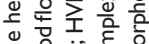

응 훙

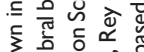

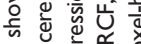

है

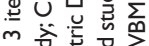

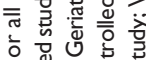

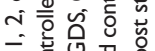

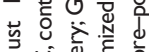

उ艾要

온 웜 등

入े 离

ᄃ

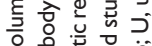

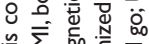

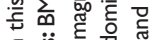

$\subseteq$ 등

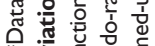




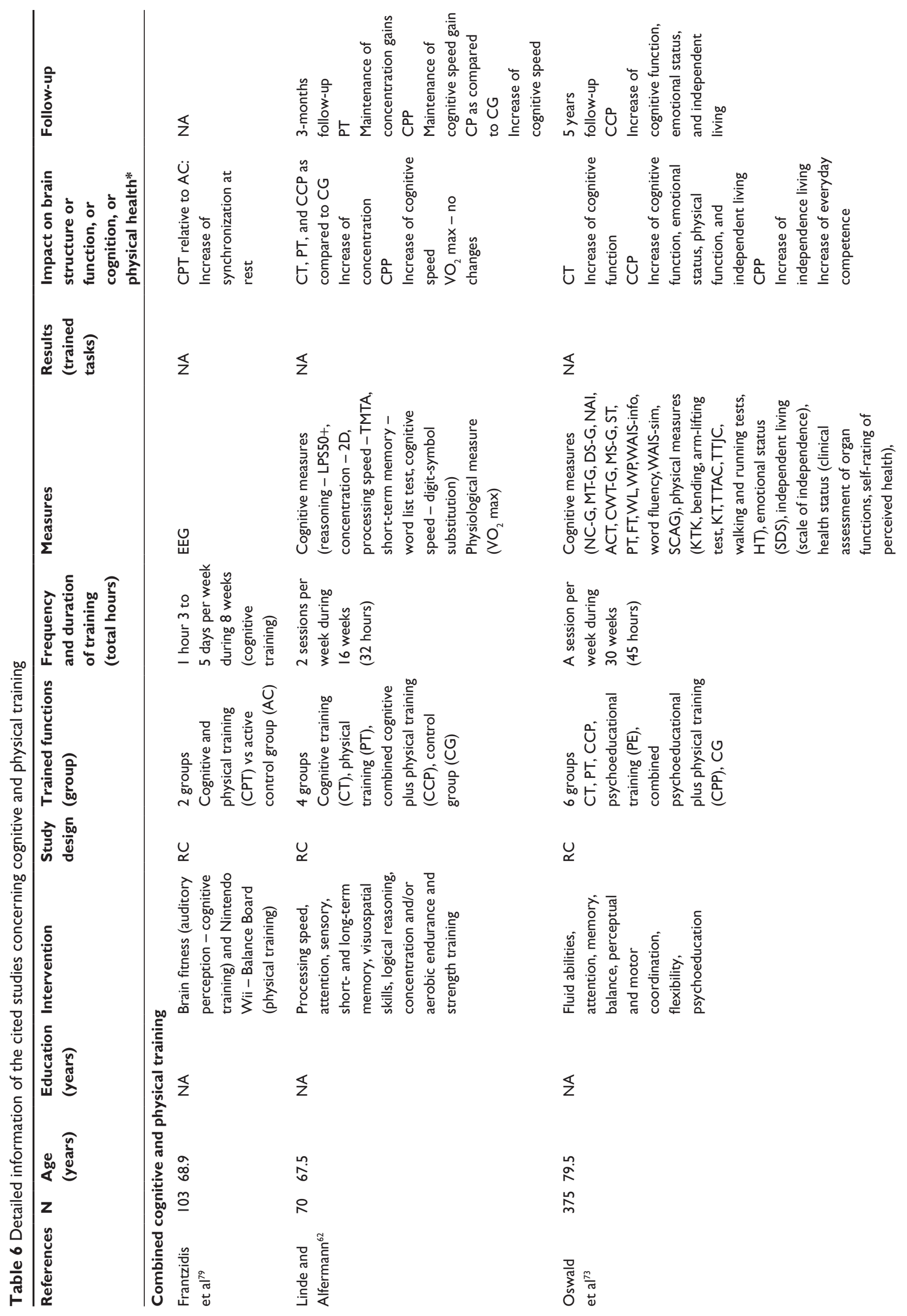




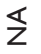

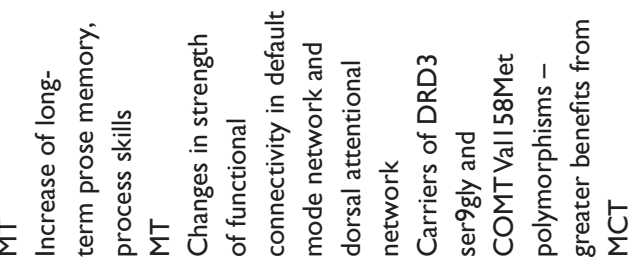

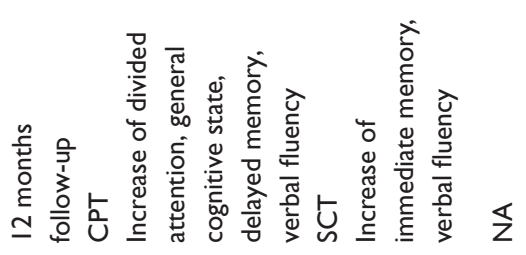

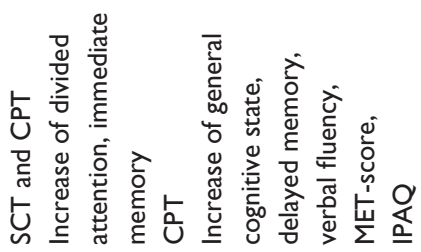

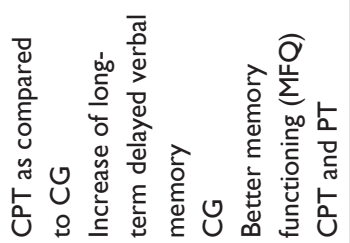

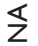
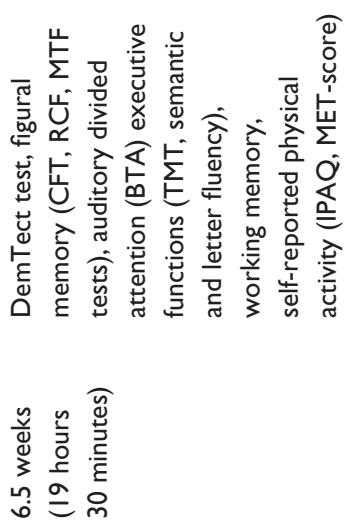

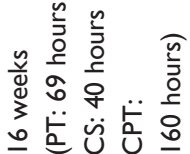
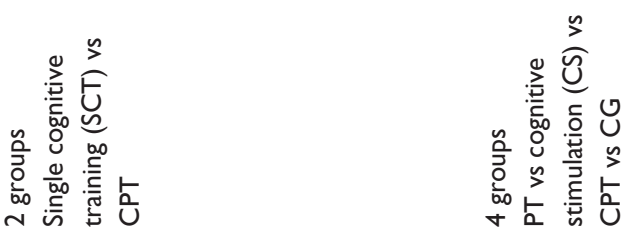

$u$
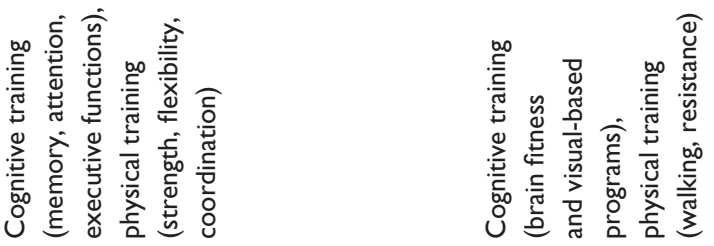

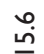

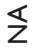

$\stackrel{m}{\circ}$

우

i̊.

$\overline{\underline{a}}$

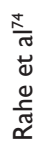

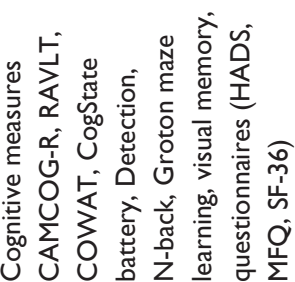

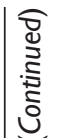

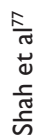

ㅇ

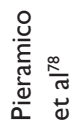




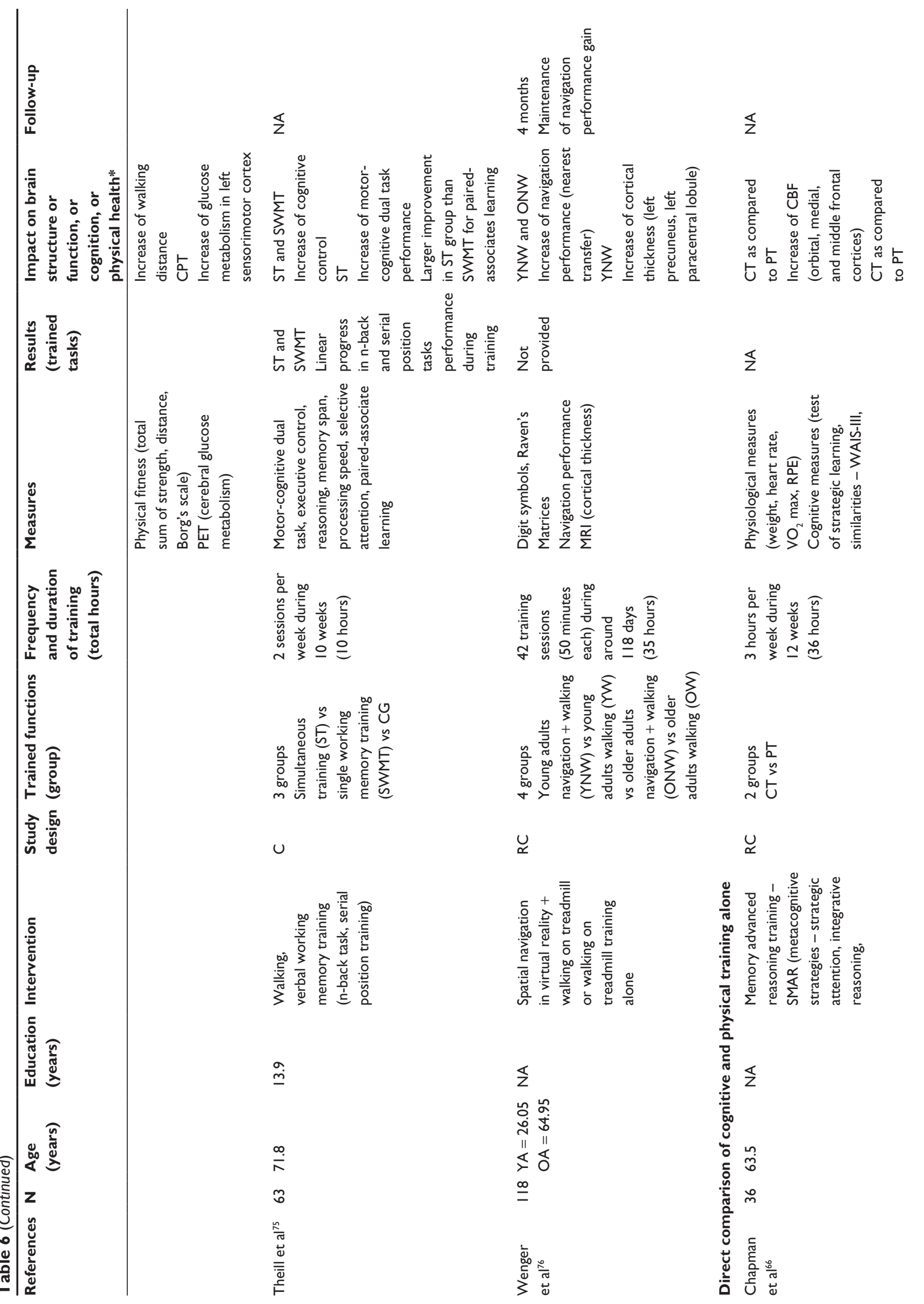



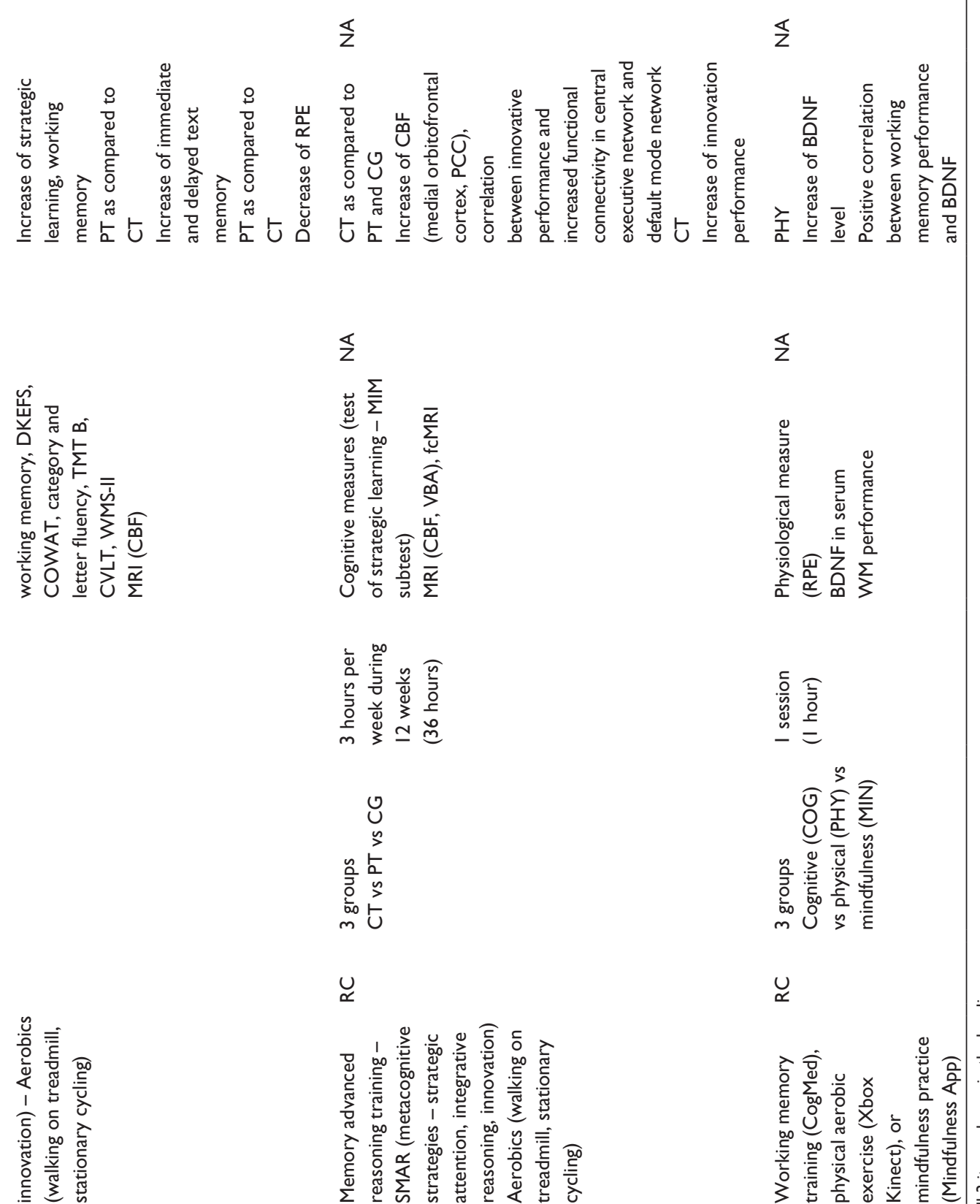

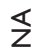

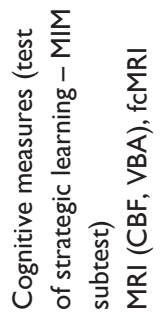

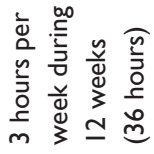

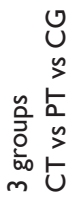

U

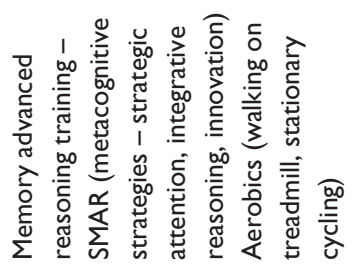

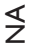

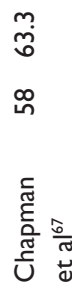

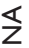

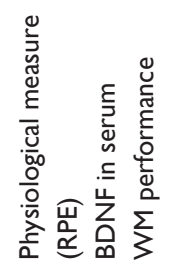

흠

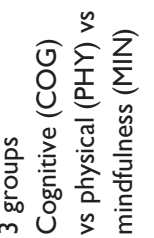

Ч

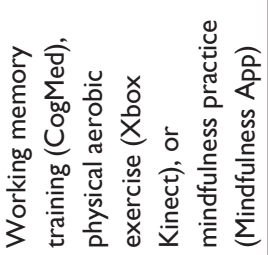

$\infty$

$\stackrel{\infty}{\circ}$

m

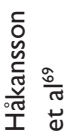

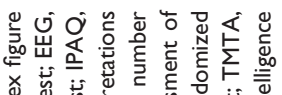

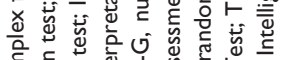

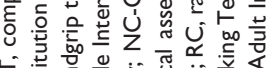

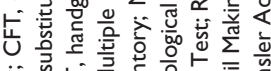

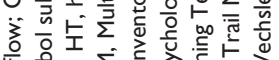

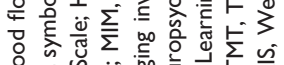

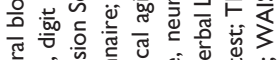

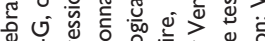

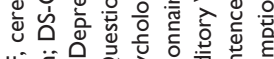

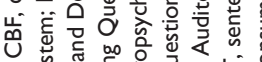

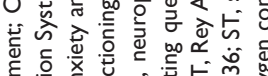

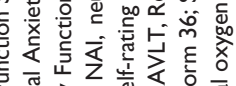

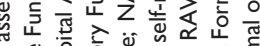

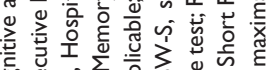

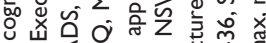

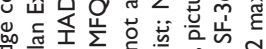

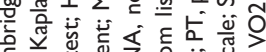

U

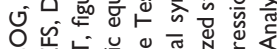

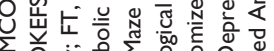

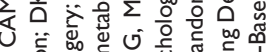

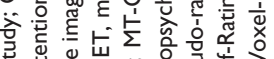

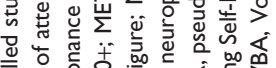

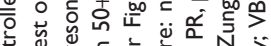

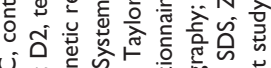

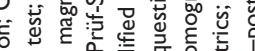

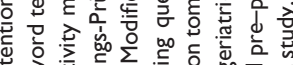

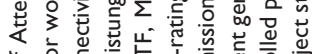

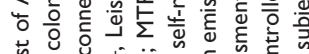

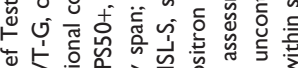

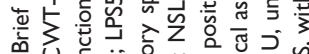

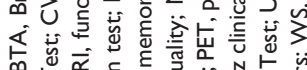

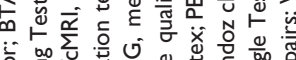

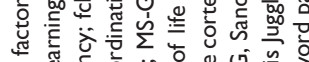

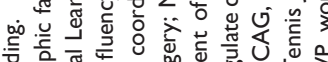

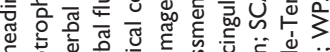

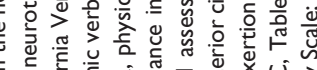

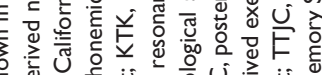

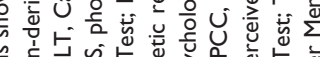

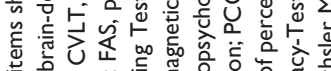

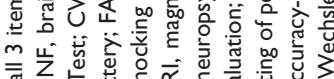

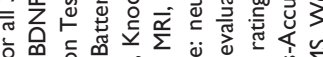

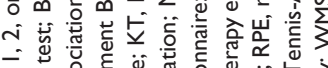

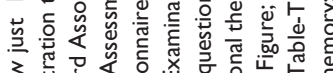

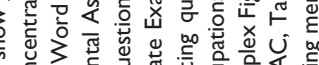

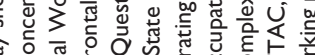

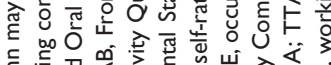

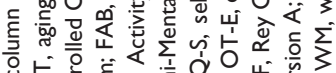

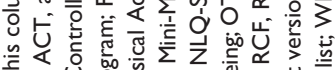

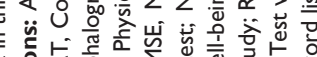

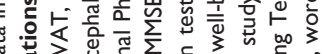

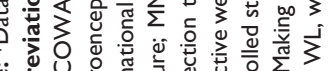

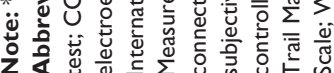


(thalamus, striatum). However, some studies did not report changes on brain level due to cognitive training. ${ }^{16,36,39}$

\section{Transfer to untrained tasks and maintenance}

Two critical issues making it possible to judge the efficiency of cognitive training are transfer and maintenance. If training is transferred to untrained tasks, then this provides evidence that the training improves cognition in a more general way. Several studies mention transfer to nearest, near, and far abilities relative to the trained tasks. However, it is not clear as to what exactly these transfers refer. Although some authors have attempted to define these concepts, ${ }^{40}$ not all studies use these informal definitions. Maintenance corresponds to the period after the end of the training after which positive outcomes of the training are still present. Several studies have focused on these two points, although not always with the same distinction concerning transfer and not always with the same post-training period in order to determine maintenance. This makes the comparison of these studies complicated and general conclusions difficult to draw. Among the 32 studies we listed in Table 4, only one study did not report data concerning transfer and one study did not find a transfer effect. In some studies, near, ${ }^{41}$ far, ${ }^{31}$ or both transfer effects were found depending on the authors' classification. ${ }^{42}$ However, many of these studies do not explicitly state what kind of transfer was examined. The situation is at its worst regarding the long-term maintenance of training benefits. Sixteen of the studies presented in Table 4 did not examine this issue. In the remaining studies, the interval between immediate post-training evaluation and follow-up varies from 2 months ${ }^{33}$ to 10 years, ${ }^{29}$ with periods of 3,6 , and 12 months being the most frequently studied. Globally, these studies provide some evidence in favor of long-term maintenance, at least for some of the benefits of cognitive training at behavioral and brain level. However, to reach better understanding of transfer and maintenance of cognitive training outcomes, more systematic investigations are need.

Borella et al, for example, addressed the issues of transfer and maintenance in a systematic way. ${ }^{15}$ They examined nearest (visuo-spatial working memory), near (ie, short-term memory), and far transfer (ie, fluid intelligence, inhibition, speed processing) of working memory training at post-test, on the one hand, and long-term maintenance (8-month follow-up), on the other. Nearest, near, and far transfers were observed at post-test and follow-up, although at follow-up the effects of transfer were in general significantly lower than at post-test. These results suggest that benefits from working memory training may transfer to several cognitive functions, some of them, such as intelligence, being loosely related to trained task, and that this transfer may persist through time. Transfer of cognitive training outcomes to fluid intelligence in older adults was frequently observed ${ }^{28,31,43}$ but not always. ${ }^{33}$

\section{Transfer to everyday activities}

Because the crucial goal of cognitive training is to improve people's lives and preserve their autonomy, some authors have looked at the transfer of cognitive training benefits to everyday activities. Ten of the 32 reported studies addressed this issue, and only one did not report any effect of cognitive training on everyday living. Although the question is extremely important, it is not a straightforward task to draw conclusions about the transfer of training benefits to everyday life. This is partly due to the difficulty of objectively evaluating this transfer. In fact, everyday life abilities are most frequently evaluated with subjective measures, such as self-reported questionnaires (eg, Instrumental Activities of Daily Living). For example, Ball et al investigated the short and long-term impact of cognitive training in independencerelated activities on everyday life. ${ }^{44}$ These authors were involved in the ACTIVE study, which is the largest study to have investigated cognitive training and included 2,832 older adults who were followed up for over a period of 10 years. ${ }^{29}$ They examined if training one specific cognitive function memory, reasoning, or speed processing - would have benefits on cognition and everyday life. The transfer of acquired abilities to daily living was assessed via the everyday problem solving task (ie, reasoning abilities), everyday speed task (ie, rapidity of interactions in everyday life), and self-rated questionnaires such as activities of daily living, instrumental activities of daily living (IADL), and driving habits (ie, difficulties encountered in driving). Contrary to the short-term benefits of the trained abilities, Ball et al failed to show any generalization to everyday life after a 2 -year follow-up. ${ }^{44}$ However, the authors suggested that the functional decline of the participants after the 2-year follow-up was not great enough to make it possible to observe the long-term transfer of the training. In fact, Willis et al and Rebok et al reported that in the ACTIVE study, the autonomy of the participants in the training groups, as estimated by the self-reported IADL questionnaire, declined less than that of those in the control group both 5 and 10 years after the end of the training. ${ }^{29,45}$ The IMPACT (Improvement in Memory with Plasticitybased Adaptive Cognitive Training $)^{46}$ study also examined the participants' self-perception of cognitive functioning and mood in everyday life with the Cognitive Self-Report 
Questionnaire. Results showed that the participants noticed the improvement in their cognition and mood after training. Other authors have also reported an improvement in everyday life due to training, manifested in terms of reduced memory disorders, ${ }^{42}$ better estimated self-efficacy, ${ }^{47}$ better achievement of goals, and satisfaction with the achieved goal. ${ }^{48}$ One study showed correlation between increased white matter integrity in the occipito-temporal region (ventral attention network) after auditory perception training and improvement in everyday problem solving. ${ }^{35}$

\section{Physical training}

Physical training has been shown to induce health benefits and general well-being. Indeed, maintaining a good physical condition through life lowers the prevalence of some diseases like cancer, diabetes, and cardiovascular diseases, ${ }^{49,50}$ increases life comfort and autonomy; and delays dependence. ${ }^{51}$ Beyond the beneficial effects of physical training on body health, some authors have taken an interest in the impact of physical training on brain health, which in turn positively impacts cognition, without necessarily targeting one specific cognitive function (eg, memory, attention, reasoning, speed). The literature suggests that the neural mechanisms responsible for the impact of physical training on cognition are due to neurogenesis (ie, production of new neurons), angiogenesis (ie, growth of new blood vessels from preexisting ones), synaptogenesis (ie, formation of synapses between neurons), and the action of neurotrophins (proteins that support the survival, development, and functions of neurons). ${ }^{52}$ In the present review (all studies are presented in Table 5), we focused only on physical training, especially on aerobic training, which has been shown to bring about the greatest impact on the aging brain and cognition ${ }^{17,53}$ and delaying dependence in the healthy elderly, ${ }^{54}$ although other types of intervention are also used to improve cognition (physical activity, physical exercise). ${ }^{55}$ Physical training consists of targeted exercises as classified by the World Health Organization guidelines ${ }^{56}$ that involve muscle strength and/or endurance ${ }^{57}$ and usually includes aerobics (ie, capacity to perform large-muscle activity over a long period of time), balance (ie, backward walking, walking and turning around, one-leg stand), muscle-strengthening, resistance (ie, ability to produce force to overcome inertia or a load), and flexibility (ie, practice in the range of movements necessary in daily life).

Interestingly, some authors have suggested that physical training may improve cognition in the same way as cognitive training and that they can therefore be used interchangeably. ${ }^{6}$
Among the 10 studies listed in Table 5 (seven using structural and/or functional MRI), only one study did not report any significant improvement in cognition. ${ }^{58}$ However, the fact that this study was conducted with sedentary older adults aged more than 70 years may at least partly explain this result. All the other studies report effects on cognitive function, on brain structure/function, or on both. In addition, in seven studies listed in Table 6 (four using MRI, ${ }^{66,67,78,79}$ one using positron emission tomography, ${ }^{77}$ one using electroencephalogram, ${ }^{79}$ and one examining serum brain-derived neurotrophic factor $[\mathrm{BDNF}]^{69}$ ) that included groups undergoing single physical training (not combined with other kinds of training), positive effects of physical training on cognition and on brain structure or function were reported. Concerning cognition, improvements were especially observed for short-term ${ }^{59,60}$ and working memory, ${ }^{53}$ long-term immediate and delayed memory, ${ }^{60}$ attention, ${ }^{61,62}$ and executive functions. ${ }^{63}$ On the brain level: 1) structural changes have been found in both white $^{53,59}$ and gray matter, ${ }^{64,65}$ and 2) functional changes have been observed at the level of functional connectivity, ${ }^{53,59}$ $\mathrm{CBF},{ }^{60,66,67}$ and task-related pattern of blood oxygen level dependent response. ${ }^{17,68}$ Some authors ${ }^{61}$ suggested that the thickening of the gray matter might be due to an increase in the level of BDNF. This suggestion is in line with the observation that only physical training, as compared to cognitive and mindfulness training, produced an immediate increase in serum BDNF, which, in addition, was correlated with improved working memory performance. ${ }^{69}$

\section{Differences in outcomes of physical training as function of exercise type}

When two or more physical exercises were used and compared, authors have reported some differences in training outcomes. On the brain level, Voss et al showed that walking training induced greater white matter integrity in pre-frontal, parietal, and temporal regions than stretching training. ${ }^{53}$ These authors also observed a greater increase in functional connectivity in the default network and frontal executive network after aerobic training than after stretching and toning training after 12 months of training. Voss et al also reported a greater increase in white matter integrity in the default mode network and frontal executive network after walking training than after stretching training. ${ }^{59}$ An increase in the hippocampal volume after walking training, but not stretching, was also observed. ${ }^{65}$ Colcombe et al showed increased Flanker task-related activity in the attentional network (superior and medial frontal gyrus and superior parietal gyrus) after aerobic training but not after stretching 
training. ${ }^{17}$ Surprisingly, for the same task, Voelcker-Rahage et al reported a decrease in activity after aerobic training in several cortical areas, especially in the superior and medial frontal gyrus. ${ }^{68}$ On the contrary, they reported increased Flanker task-related activity in the inferior frontal gyrus, thalamus, caudate, and superior parietal lobule after coordination training. These findings provide evidence that not every type of aerobic training induces neuroplasticity to the same extent.

On the cognitive level, although no given form of physical training (ie, aerobics, running, muscle building) is thought to specifically improve any particular cognitive function, some studies have pointed out that the outcomes of physical training for cognition may depend on the type of physical activity used in the training. For example, Colcombe et al and Liu-Ambrose et al showed improvements in resistance to interference after aerobic training, ${ }^{17,63}$ and Voss et al found an improvement in working memory. ${ }^{59}$ However, working memory improvement has also been observed after resistance training. ${ }^{70}$ These results suggest that combining different types of activity in physical training sessions may well be a better way to train because these combined activities act on more than one aspect of cognitive function while, at the same time, different types of training may act on one and the same function and potentially reinforce the benefits. Combining different types of physical training would therefore be more efficient than using only a single type. However, Voss et al investigated the impact of aerobic versus stretching training on short-term memory, working memory, and executive control and showed that working memory was enhanced only in the walking training group. ${ }^{53}$ Stretching training did not lead to any working memory improvement, suggesting that not all types of physical training have the same impact on the same cognitive function. Some authors consider that aerobic training is the most efficient in terms of cognitive enhancement and its impact is thought to be mediated by cardiovascular fitness. ${ }^{53}$

\section{Equivalence between physical training and physical activity through life}

One interesting question related to physical training is whether the training outcomes on cognition are equivalent to or different from those produced by a high level of physical activity through life. This point was addressed by Colcombe et al. ${ }^{17}$ These authors observed that, after 6 months, older adults who took part in aerobic training (study 2) achieved a high level of resistance to interference that was associated with reduced activity in the anterior cingulate cortex.
Interestingly, the same pattern of results was observed in non-trained older adults with a high level of aerobic fitness (study 1). These results suggest that the neurocognitive benefits of cardiovascular fitness may come both from a physically active life that leads to physical fitness and from physical training. This is an important argument in favor of physical training in older adults.

\section{Transfer to everyday activities}

Generally, the transfer of physical training outcomes to everyday life was not evaluated in the studies we included in the present review. Only Liu-Ambrose et al reported that physical training reduced falls in everyday life and that this reduction was linked to improvement of executive function. ${ }^{63}$

\section{Summary of findings for cognitive and physical training}

To summarize, both cognitive and physical training seem to induce changes in brain and cognition of healthy older adults. However, studies exploring physical training outcomes have seldom examined long-term maintenance and transfer to everyday life. Thus, it seems important for future studies to address this shortcoming. Concerning short-term changes at brain level, both trainings seem to have some positive outcomes for the structure and function of older adults' brains, although these changes have more frequently been examined in studies focusing on physical training. Both types of training seem to impact frontal lobe functioning and increase white matter integrity in frontal and parietal regions, with physical training being more frequently reported to confer such benefits. By contrast, as far as the hippocampus is concerned, structural and functional changes have been examined and reported almost exclusively by studies involving physical training. Concerning short-term changes in cognition, both training programs have been reported to impact positively on, in particular, executive functions (ie, planning, inhibition, and coordination) and memory (ie, short, long-term, and working memory). Beyond these convergent benefits, cognitive and physical training also have a differential impact on cognition. Cognitive training tends to improve problem solving and fluid intelligence, and reduce the cost of multitasking, whereas physical training tends to improve spatial memory, speed, and resistance to interference. There is no strong evidence of a systematic transfer of the benefits of training to untrained tasks and even less to everyday life activities, ie, the main aims of cognitive training. In addition, when transfer occurs, it does not seem to systematically persist through time. 


\section{Combined cognitive and physical training}

Both cognitive and physical training are used in clinical interventions, because they have been proved to have a certain effectiveness. Given that there is some evidence that they influence brain structure and function, on the one hand, and cognition, on the other, in different ways, one may hypothesize that they should bring about greater benefits for cognition when they are combined in one intervention than when either form of training is used alone.

Two systematic reviews of literature investigated this question. ${ }^{71,72}$ The authors showed that CCPT, whether simultaneous or subsequent, would produce better benefits on cognition as compared to cognitive and physical training alone. However, these reviews point out two issues: the impact of cognitive training seems to be limited to trained functions ${ }^{71}$ and the benefits of CCPT seem to be difficult to evaluate in populations with cognitive impairment. ${ }^{72}$ In fact, these two reviews put the emphasis on the impact of CCPT on healthy versus cognitively impaired populations, whereas the present review is entirely focused on the comparison of impact of single versus combined training on healthy older adult's brain and cognition.

Only a few empirical studies have systematically investigated this question. To our knowledge, only four behavioral studies ${ }^{62,73-75}$ and two neuroimaging studies ${ }^{76,77}$ have directly compared single versus combined training and their impact on cognition in healthy older adults (all cited studies concerning CCPT are shown in Table 6). In addition, two studies have compared combined training with a control group only, ${ }^{78,79}$ and three studies have compared single physical training with single cognitive training and a control group. ${ }^{66,67,69}$

\section{Methodological issues for combined cognitive and physical training}

Before we address the effectiveness and potential advantage of CCPT over single physical and single cognitive training, it is necessary to point out an important methodological question. Indeed, the organization of protocols in which physical and cognitive training are combined raises the question of whether the training modes should be sequential or simultaneous. In the studies listed in Table 6, two studies used simultaneous training, ${ }^{75,76}$ performing a cognitive task while walking on a treadmill. Both sequential and simultaneous CCPT may have their advantages and drawbacks. The advantage of sequential training is that individuals can be entirely focused on the current training. However, in the sense that cognitive and physical parts of the training are not performed at the same time, there is no direct possible interaction between the mechanisms underpinning each training session. Conversely, although simultaneous training permits an interaction between these mechanisms, the fact that individuals are confronted with a dual task might mean that they do not focus fully on all aspects of each training session. Is it possible to run and simultaneously perform cognitive training efficiently? This point is a matter of debate in the literature. Indeed, it has been shown that dual tasks are particularly difficult for older adults. Consequently, multitasking is very sensitive to aging, and the cost of performing a dual task increases as people age ${ }^{80}$ However, Theill et al showed that older adults do not find it any more difficult to follow CCPT (ie, performing cognitive tasks while walking on a treadmill) than to undertake single cognitive training. They argued that the mobilization of multiple resources and abilities corresponds to the way that people act during their everyday activities. ${ }^{75}$ The question of sequential or simultaneous training also raises the issue of the duration of the training. Indeed, in the case of sequential training, the difficulty lies in deciding whether it is better to keep the same training duration as for single training (eg, 1 hour per week), but to dedicate only half this time to each component (eg, 30 minutes per week of cognitive training and 30 minutes per week of physical training), or to double the overall training time in order to keep the same training time for each type of training (eg, 1 hour per week of cognitive training and 1 hour per week of physical training) as compared to their respective single training session times. In the latter case, it is not clear whether any advantage observed for combined training is due to the combination of the training types or simply to the fact that training lasts for twice as long. It is therefore possible that the design of the training may influence its outcomes, although, as far as we know, no studies have directly compared sequential training with simultaneous training.

\section{Advantage of combined cognitive and physical training Short-term outcomes on cognition and brain}

Among the five studies that directly compared CCPT with single physical or cognitive training on short-term outcomes, all observed an advantage of combined training. Oswald et al showed positive specific outcomes after cognitive training alone, combined physical and psychoeducational training, and CCPT but not after physical training alone. ${ }^{73}$ However, as compared to control group, CCPT brought the largest gains in cognitive functioning, emotional status, and physical 
functioning. Theill et al compared groups receiving CCPT (ie, simultaneous working memory and cardiovascular training) or single training (ie, working memory) with a control group. They reported an improvement in executive control after both types of training, whereas only the CCPT group exhibited improved performance in a motor-cognitive dual task and also achieved greater gains in paired-associates learning. These results suggest that CCPT may produce both similar and specific outcomes to single physical and cognitive training. ${ }^{75}$ Two other studies confirm this suggestion. Linde and Alfermann reported that, compared to a control group, sequential CCPT and single cognitive and physical training increased concentration, but that only CCPT improved cognitive speed. ${ }^{62}$ Rahe et al found that CCPT and single cognitive training improved divided attention and immediate memory, whereas CCPT additionally improved general cognitive state, delayed memory, and verbal fluency. However, there was no control group in this study. ${ }^{74}$ This was also the case in the study by Wenger et al where simultaneous CCPT training was reported to produce greater improvement in spatial navigation performance than single physical training, but neither of these training types resulted in cortical thickening. ${ }^{76}$ Finally, Shah et al reported a greater improvement in longterm verbal memory after sequential CCPT than in a control group. In addition, the CCPT group presented increased glucose metabolism in the left sensorimotor cortex, and this was correlated with better performance in the memory task. There was no advantage of single physical or cognitive training on cognition. ${ }^{77}$

Globally, the CCPT training seems to result in greater short-term benefits than single training, whether cognitive or physical. However, the results have to be treated with caution because in the reported studies, the sequential CCPT took twice the amount of time as the single training program. Thus, the greater benefits at both the behavioral and brain level observed after this training could be due to reasons other than the simple combination of cognitive and physical training, such as a superior training time.

In addition, positive outcomes of CCPT were shown in two studies that compared CCPT to a control group only. Frantzidis et al reported increased frontoparietal synchronization at rest after CCPT as compared to a physically active control group. Unfortunately, they did not report behavioral outcomes. ${ }^{79}$ Pieramico et al observed an improvement in long-term memory and processing skills due to CCPT as compared to a passive control group. ${ }^{78}$ Changes in the strength of the functional connectivity of the default mode and dorsal attentional networks were also observed.
In addition, carriers of DRD3 ser9gly and COMT Val158Met polymorphism of dopamine-related genes benefited more from CCPT.

\section{Long-term maintenance}

The question of the long-term maintenance of benefits due to CCPT was addressed by four studies. All of these have shown the persistence of benefits: at 5-year follow-up, ${ }^{73}$ at 1-year follow-up, ${ }^{74}$ at 4-month follow-up, ${ }^{76}$ and at 3-month follow-up. ${ }^{62}$ Oswald et al observed, 4 years after the end of the training, improvement of cognitive functioning after both cognitive training alone and CCPT, with the largest improvement after CCPT. Importantly, the participants from CCPT group expressed higher degree of independent living, and the symptoms of cognitive impairment were less pronounced among the members of the CCPT group than control group. ${ }^{73}$ In the Linde and Alfermann's study, the benefits due to single training were also found to be maintained, suggesting that there is probably no specific mechanism responsible for maintaining the benefits of CCPT. ${ }^{62}$ In Rahe et al's study, an improvement in divided attention was observed immediately after the end of single cognitive training and CCPT, but only the CCPT group showed further improvement at 1-year follow-up. ${ }^{74}$ The authors suggested that physical training might enhance brain metabolism and plasticity, whereas cognitive training, by increasing mental demand, might use and reinforce the enhanced brain metabolism and guide brain plasticity. In other words, physical and cognitive training play a different but complementary role in brain plasticity. Physical training can be seen as an initiator of brain plasticity, while cognitive training would then subsequently reinforce the direction introduced by physical training. These results suggest that CCPT makes a robust contribution to slowing down attentional deficit in aging over the long term.

\section{Transfer to everyday activities}

Generally, the transfer of CCPT outcomes to everyday life was not evaluated in the studies we included in the present review. Only Oswald et al reported that this training has a positive impact on independent living and every day competence at short term and only on independent living at long term (5 years).

\section{Conclusion and recommendations}

The main aim of this review was to examine the potential advantage of combining cognitive and physical training into one intervention in older adults in terms of both immediate 
and long-term benefits on cognition and everyday life. It seems very clear that cognitive and physical training both have positive outcomes for brain structure and function, as well as in terms of improved cognition. In addition, some studies are beginning to show a positive impact of training on the autonomy and quality of everyday life of elderly people. However, too little studies examined this point and clearly future studies should seriously investigate weather and to what extent cognitive and physical training improves older people's everyday life. Especially, there is a cruel lack of data concerning physical training. Importantly, some evidence suggests that cognitive and physical training may complement one another and help improve both brain structure and function, and cognition. Unfortunately, here again too little studies compared in a systematic way CCPT with each of these training sessions administered alone. Thus, although the conclusions of these few studies indicate an advantage for combined training, further studies are necessary to be able to draw more robust conclusion in favor of this training. However, at the level of clinical application, although the experimental data are too few and not always unequivocal, it seems preferable to consider these two training types when planning interventions designed to improve cognitive capacities in older people. The role of future studies will be to provide more evidence in favor of advantage of combined training and to investigate methodological issues inherent to the design of CCPT in order to determine the most efficient protocols.

\section{Acknowledgments}

We thank Tim Pownall, native English speaker, for English editing. This work was supported by the LabEx Cortex (ANR-11-LABX-0042) of Universite de Lyon, within the program “Investissements d'Avenir" (ANR-11-IDEX-0007) operated by the French National Research Agency (Agence Nationale de la Recherche).

\section{Disclosure}

The authors report no conflicts of interest in this work.

\section{References}

1. World Health Organization. Active Ageing: A Policy Framework. Geneva, Switzerland: World Health Organization; 2002.

2. Aromaa A. Health and Functional Capacity in Finland: Baseline Results of the Health 2000 Health Examination Survey. Kansanterveyslaitos: National Public Health Institute; 2004. Consulté à l'adresse. Available from: http://www.julkari.fi/handle/10024/78534. Accessed February 1, 2018.

3. Stenzelius K, Westergren A, Thorneman G, Hallberg IR. Patterns of health complaints among people $75+$ in relation to quality of life and need of help. Arch Gerontol Geriat. 2005;40(1):85-102.
4. Manton KG, Land KC. Active life expectancy estimates for the U.S. elderly population: A multidimensional continuous-mixture model of functional change applied to completed Cohorts, 1982-1996. Demography. 2000; 37(3):253-265.

5. Penninx BWJH, Rejeski WJ, Pandya J, et al. Exercise and depressive symptoms: a comparison of aerobic and resistance exercise effects on emotional and physical function in older persons with high and low depressive symptomatology. J Gerontol B. 2002;57(2):P124-P132.

6. Kramer AF, Erickson KI. Capitalizing on cortical plasticity: influence of physical activity on cognition and brain function. Trends Cogn Sci. 2007;11(8):342-348.

7. Reuter-Lorenz PA, Park DC. How Does it STAC Up? Revisiting the scaffolding theory of aging and cognition. Neuropsychol Rev. 2014; 24(3):355-370.

8. Ska B, Joanette Y. Vieillissement normal et cognition. Med Sci. 2006; 22(3):284-287.

9. Gunning-Dixon FM, Gur RC, Perkins AC, et al. Age-related differences in brain activation during emotional face processing. Neurobiol Aging. 2003;24(2):285-295.

10. Cabeza R. Hemispheric asymmetry reduction in older adults: the HAROLD model. Psychol Aging. 2002;17(1):85-100.

11. Reuter-Lorenz PA, Lustig C. Brain aging: reorganizing discoveries about the aging mind. Curr Opin Neurobiol. 2005;15(2):245-251.

12. Li S-C, Lindenberger U. Cross-level unification: a computational exploration of the link between deterioration of neurotransmitter systems and dedifferentiation of cognitive abilities in old age. In: Nilsson L-G, Markowitsch HJ, editors. Cognitive Neuroscience of Memory. Seattle, WA: Hogrefe and Huber Publishers; 1999:103-146.

13. Bier B, Belleville S. Optimiser le fonctionnement cognitif au cours du vieillissement: facteurs de réserve, stimulation cognitive et plasticité cérébrale [Optimizing cognition in aging: brain reserve, cognitive stimulation and cerebral plasticity]. Neuropsicologia Latinoamericana. 2010;2(3):37-47. French.

14. Zahodne LB, Stern Y, Manly JJ. Differing effects of education on cognitive decline in diverse elders with low versus high educational attainment. Neuropsychology. 2015;29(4):649-657.

15. Borella E, Carretti B, Riboldi F, De Beni R. Working memory training in older adults: evidence of transfer and maintenance effects. Psychol Aging. 2010;25(4):767-778.

16. Cao X, Yao Y, Li T, et al. The impact of cognitive training on cerebral white matter in community-dwelling elderly: one-year prospective longitudinal diffusion tensor imaging study. Sci Rep. 2016;6:33212.

17. Colcombe SJ, Kramer AF, Erickson KI, et al. Cardiovascular fitness, cortical plasticity, and aging. Proc Natl Acad Sci U S A. 2004;101(9): 3316-3321.

18. Maher CG, Sherrington C, Herbert RD, Moseley AM, Elkins M. Reliability of the PEDro scale for rating quality of randomized controlled trials. Phys Ther. 2003;83(8):713-721.

19. Goh JO, Park DC. Neuroplasticity and cognitive aging: the scaffolding theory of aging and cognition. Resto Neurol Neuros. 2009;27(5): 391-403.

20. Chiu H-L, Chu H, Tsai J-C, et al. The effect of cognitive-based training for the healthy older people: a meta-analysis of randomized controlled trials. PLoS One. 2017;12(5):e0176742.

21. Kelly ME, Loughrey D, Lawlor BA, Robertson IH, Walsh C, Brennan S. The impact of cognitive training and mental stimulation on cognitive and everyday functioning of healthy older adults: a systematic review and meta-analysis. Ageing Res Rev. 2014;15:28-43.

22. Martin M, Clare L, Altgassen AM, Cameron MH, Zehnder F. Cognitionbased interventions for healthy older people and people with mild cognitive impairment. Cochrane Database Syst Rev. 2011;(1):Cd006220.

23. Hindin SB, Zelinski EM. Extended practice and aerobic exercise interventions benefit untrained cognitive outcomes in older adults: a meta-analysis. J Am Geriatr Soc. 2012;60(1):136-141.

24. van Uffelen JG, Chin APMJ, Hopman-Rock M, van Mechelen W. The effects of exercise on cognition in older adults with and without cognitive decline: a systematic review. Clin J Sport Med. 2008;18(6):486-500. 
25. Young J, Angevaren M, Rusted J, Tabet N. Aerobic exercise to improve cognitive function in older people without known cognitive impairment. Cochrane Database Syst Rev. 2015;(4):Cd005381.

26. La Rue A. Healthy brain aging: role of cognitive reserve, cognitive stimulation, and cognitive exercises. Clin Geriatr Med. 2010;26(1):99-111.

27. Kueider A, Bichay K, Rebok GW. Cognitive Training for Older Adults: What Is It and Does It Work? Washington, DC: American Institutes for Research; 2014.

28. Basak C, Boot WR, Voss MW, Kramer AF. Can training in a real-time strategy video game attenuate cognitive decline in older adults? Psychol Aging. 2008;23(4):765-777.

29. Rebok GW, Ball K, Guey LT; ACTIVE Study Group. Ten-year effects of the advanced cognitive training for independent and vital elderly cognitive training trial on cognition and everyday functioning in older adults. J Am Geriatr Soc. 2014;62(1):16-24.

30. Schmiedek F, Lövdén M, Lindenberger U. Hundred days of cognitive training enhance broad cognitive abilities in adulthood: findings from the COGITO Study. Front Aging Neurosci. 2010;2:27.

31. Karbach J, Kray J. How useful is executive control training? Age differences in near and far transfer of task-switching training. Dev Sci. 2009; 12(6):978-990.

32. Anguera JA, Boccanfuso J, Rintoul JL, et al. Video game training enhances cognitive control in older adults. Nature. 2013;501(7465):97-101.

33. Ackerman PL, Kanfer R, Calderwood C. Use it or lose it? Wii brain exercise practice and reading for domain knowledge. Psychol Aging. 2010;25(4):753-766.

34. Chapman SB, Aslan S, Spence JS, et al. Neural mechanisms of brain plasticity with complex cognitive training in healthy seniors. Cereb Cortex. 2015;25(2):396-405.

35. Strenziok M, Parasuraman R, Clarke E, Cisler DS, Thompson JC, Greenwood PM. Neurocognitive enhancement in older adults: comparison of three cognitive training tasks to test a hypothesis of training transfer in brain connectivity. NeuroImage. 2014;85(Part 3):1027-1039.

36. Mozolic JL, Hayaska S, Laurienti PJ. A cognitive training intervention increases resting cerebral blood flow in healthy older adults. Front Hum Neurosci. 2010;4:16.

37. Dahlin E, Neely AS, Larsson A, Bäckman L, Nyberg L. Transfer of learning after updating training mediated by the striatum. Science. 2008;320(5882):1510-1512.

38. Berry AS, Zanto TP, Clapp WC, et al. The influence of perceptual training on working memory in older adults. PLoS One. 2010;5(7):e11537.

39. Heinzel S, Schulte S, Onken J, et al. Working memory training improvements and gains in non-trained cognitive tasks in young and older adults. Neuropsychol Dev Cogn B Aging Neuropsychol Cogn. 2014; 21(2):146-173.

40. Willis SL. Methodological issues in behavioral intervention research with the elderly. In: Birren JE, Schaie KW, editors. Handbook of the Psychology of Aging. San Diego, CA: Academic Press; 2001:78-108.

41. Li S-C, Schmiedek F, Huxhold O, Röcke C, Smith J, Lindenberger U. Working memory plasticity in old age: practice gain, transfer, and maintenance. Psychol Aging. 2008;23(4):731-742.

42. Brehmer Y, Westerberg H, Bäckman L. Working-memory training in younger and older adults: training gains, transfer, and maintenance. Front Hum Neurosci. 2012;6:63.

43. Stine-Morrow EAL, Parisi JM, Morrow DG, Park DC. The effects of an engaged lifestyle on cognitive vitality: a field experiment. Psychol Aging. 2008;23(4):778-786.

44. Ball K, Berch DB, Helmers KF, et al. Effects of cognitive training interventions with older adults: a randomized controlled trial. JAMA. 2002;288(18):2271-2281.

45. Willis SL, Tennstedt SL, Marsiske M, et al. Long-term effects of cognitive training on everyday functional outcomes in older adults. JAMA. 2006;296(23):2805-2814

46. Smith GE, Housen $\mathrm{P}$, Yaffe K, et al. A cognitive training program based on principles of brain plasticity: results from the Improvement in Memory with Plasticity-based Adaptive Cognitive Training (IMPACT) Study. J Am Geriat Soc. 2009;57(4):594-603.
47. Cavallini E, Pagnin A, Vecchi T. Aging and everyday memory: the beneficial effect of memory training. Arch Gerontol Geriat. 2003;37(3): 241-257.

48. McAvinue LP, Golemme M, Castorina M, et al. An evaluation of a working memory training scheme in older adults. Front Aging Neurosci. 2013;5:20.

49. Booth FW, Gordon SE, Carlson CJ, Hamilton MT. Waging war on modern chronic diseases: primary prevention through exercise biology. J Appl Physiol. 2000;88(2):774-787.

50. Myers J, Prakash M, Froelicher V, Do D, Partington S, Atwood JE. Exercise capacity and mortality among men referred for exercise testing. New Engl J Med. 2002;346(11):793-801.

51. Liubicich ME, Magistro D, Candela F, Rabaglietti E, Ciairano S. Physical activity and mobility function in elderly people living in residential care facilities. "Act on Aging": a pilot study. Adv Phy Educ. 2012;2(2):54.

52. van Praag H. Exercise and the brain: something to chew on. Trends Neurosci. 2009;32(5):283-290.

53. Voss MW, Heo S, Prakash RS, et al. The influence of aerobic fitness on cerebral white matter integrity and cognitive function in older adults: results of a one-year exercise intervention. Hum Brain Mapp. 2013; 34(11):2972-2985.

54. Guilley E, Ghisletta P, Armi F, et al. Dynamics of Frailty and ADL. Dependence in a Five-Year Longitudinal Study of Octogenarians. Res Aging. 2008;30:299-317.

55. Caspersen CJ, Powell KE, Christenson GM. Physical activity, exercise, and physical fitness: definitions and distinctions for health-related research. Public Health Rep. 1985;100(2):126-131.

56. WHO. Making a difference. The World Health Report 1999. Health Millions. 1999;25(4):3-5.

57. Rydwik E, Frändin K, Akner G. Effects of physical training on physical performance in institutionalised elderly patients (70+) with multiple diagnoses. Age Ageing. 2004;33(1):13-23.

58. Sink KM, Espeland MA, Castro CM, et al. Effect of a 24-month physical activity intervention vs health education on cognitive outcomes in sedentary older adults: the LIFE randomized trial. JAMA. 2015; 314(8):781-790.

59. Voss MW, Prakash RS, Erickson KI, et al. Plasticity of brain networks in a randomized intervention trial of exercise training in older adults. Fron Aging Neurosci. 2010;2:32.

60. Chapman SB, Aslan S, Spence JS, et al. Shorter term aerobic exercise improves brain, cognition, and cardiovascular fitness in aging. Front Aging Neurosci. 2013;5:75.

61. Liu-Ambrose T, Nagamatsu LS, Graf P, Beattie BL, Ashe MC, Handy C. Resistance training and executive functions: a 12-month randomized controlled trial. Arch Int Med. 2010;170(2):170-178.

62. Linde K, Alfermann D. Single versus combined cognitive and physical activity effects on fluid cognitive abilities of healthy older adults: a 4-month randomized controlled trial with follow-up. J Aging Phys Act. 2014;22(3):302-313.

63. Liu-Ambrose T, Donaldson MG, Ahamed Y, et al. Otago home-based strength and balance retraining improves executive functioning in older fallers: a randomized controlled trial. J Am Geriat Soc. 2008; 56(10):1821-1830.

64. Boyke J, Driemeyer J, Gaser C, Büchel C, May A. Training-induced brain structure changes in the elderly. J Neurosci. 2008;28(28):7031-7035.

65. Erickson KI, Voss MW, Prakash RS, et al. Exercise training increases size of hippocampus and improves memory. Proc Natl Acad Sci. 2011; 108(7):3017-3022.

66. Chapman SB, Aslan S, Spence JS, et al. Distinct brain and behavioral benefits from cognitive vs. physical training: a randomized trial in aging adults. Front Hum Neurosci. 2016;10:338.

67. Chapman SB, Spence JS, Aslan S, Keebler MW. Enhancing innovation and underlying neural mechanisms via cognitive training in healthy older adults. Front Aging Neurosci. 2017;9:314.

68. Voelcker-Rehage C, Godde B, Staudinger UM. Cardiovascular and coordination training differentially improve cognitive performance and neural processing in older adults. Front Hum Neurosci. 2011;5:26. 
69. Håkansson K, Ledreux A, Daffner K, et al. BDNF responses in healthy older persons to 35 minutes of physical exercise, cognitive training, and mindfulness: associations with working memory function. J Alzheimers Dis. 2017;55(2):645-657.

70. Cassilhas RC, Viana VAR, Grassmann V, et al. The impact of resistance exercise on the cognitive function of the elderly. Med Sci Sports Exerc. 2007;39(8):1401-1407.

71. Lauenroth A, Ioannidis AE, Teichmann B. Influence of combined physical and cognitive training on cognition: a systematic review. $B M C$ Geriatr. 2016;16:141

72. Law LLF, Barnett F, Yau MK, Gray MA. Effects of combined cognitive and exercise interventions on cognition in older adults with and without cognitive impairment: a systematic review. Ageing Res Rev. 2014; 15:61-75.

73. Oswald WD, Gunzelmann T, Rupprecht R, Hagen B. Differential effects of single versus combined cognitive and physical training with older adults: the SimA study in a 5-year perspective. Eur J Ageing. 2006; 3(4):179.

74. Rahe J, Petrelli A, Kaesberg S, Fink GR, Kessler J, Kalbe E. Effects of cognitive training with additional physical activity compared to pure cognitive training in healthy older adults. Clin Interv Aging. 2015;10:297.

75. Theill N, Schumacher V, Adelsberger R, Martin M, Jäncke L. Effects of simultaneously performed cognitive and physical training in older adults. BMC Neurosci. 2013;14:103.

76. Wenger E, Schaefer S, Noack H, et al. Cortical thickness changes following spatial navigation training in adulthood and aging. NeuroImage. 2012;59(4):3389-3397.

77. Shah T, Verdile G, Sohrabi H, et al. A combination of physical activity and computerized brain training improves verbal memory and increases cerebral glucose metabolism in the elderly. Transl Psychiatry. 2014; 4(12):e487.

78. Pieramico V, Esposito R, Sensi F. Combination training in aging individuals modifies functional connectivity and cognition, and is potentially affected by dopamine-related genes. PLoS One. 2012; 7(8):e43901.

79. Frantzidis CA, Ladas A-KI, Vivas AB, Tsolaki M, Bamidis PD. Cognitive and physical training for the elderly: Evaluating outcome efficacy by means of neurophysiological synchronization. Int J Psychophysiol 2014;93(1):1-11.
80. Verhaeghen P. Aging and executive control: reports of a demise greatly exaggerated. Curr Dir Psychol Sci. 2011;20(3):174-180.

81. Buschkuehl M, Jaeggi SM, Hutchison S, et al. Impact of working memory training on memory performance in old-old adults. Psychol Aging. 2008;23(4):743-753.

82. Cheng Y, Wu W, Feng W, et al. The effects of multi-domain versus single-domain cognitive training in non-demented older people: a randomized controlled trial. BMC Med. 2012;10:30.

83. Mahncke HW, Connor BB, Appelman J, et al. Memory enhancement in healthy older adults using a brain plasticity-based training program: A randomized, controlled study. PNAS. 2006;103(33):12523-12528.

84. Richmond LL, Morrison AB, Chein JM, Olson IR. Working memory training and transfer in older adults. Psychol Aging. 2011;26(4): 813-822.

85. Stepankova H, Lukavsky J, Buschkueh, M, Kopecek M, Ripova D, Jaeggi SM. The malleability of working memory and visuospatial skills: a randomized controlled study in older adults. Dev Psychol. 2014; 50(4):1049-1059.

86. von Bastian CC, Langer N, Jäncke L, Oberauer K. Effects of working memory training in young and old adults. Mem Cognit. 2013;41(4):611-624.

87. Wolinsky FD, Vander Weg MW, Martin R, et al. Does cognitive training improve internal locus of control among older adults? J Gerontol B Psychol Sci Soc Sci. 2010;65(5):591-598.

88. Zinke K, Zeintl M, Rose NS, Putzmann J, Pydde A, Kliegel M. Working memory training and transfer in older adults: effects of age, baseline performance, and training gains. Dev Psychol. 2014;50(1): 304-315.

89. Lachman ME, Baltes P, Nesselroade J, Willis SL. Examination of personality-ability relationships in the elderly: The role of contextual (interface) assessment mode. J Res Pers. 1982;16(4):485-501.

90. Dahlin E, Nyberg L, Bäckman L, Neely AS. Plasticity of executive functioning in young and older adults: immediate training gains, transfer, and long-term maintenance. Psychology and Aging. 2008;23(4):720-730.

91. Mozolic JL, Long AB, Morgan AR, Rawley-Payne M, Laurienti PJ. A cognitive training intervention improves modality-specific attention in a randomized controlled trial of healthy older adults. Neurobiology of Aging. 2011;32(4):655-668.
Clinical Interventions in Aging

\section{Publish your work in this journal}

Clinical Interventions in Aging is an international, peer-reviewed journal focusing on evidence-based reports on the value or lack thereof of treatments intended to prevent or delay the onset of maladaptive correlates of aging in human beings. This journal is indexed on PubMed Central, MedLine,

\section{Dovepress}

CAS, Scopus and the Elsevier Bibliographic databases. The manuscript management system is completely online and includes a very quick and fair peer-review system, which is all easy to use. Visit http://www.dovepress. com/testimonials.php to read real quotes from published authors. 